\title{
A micromechanical model of hardening, rate sensitivity and thermal softening in bec single crystals
}

\author{
L. Stainier ${ }^{\mathrm{a}}$, A.M. Cuitiño ${ }^{\mathrm{b}}$, M. Ortiz ${ }^{\mathrm{c}, *}$ \\ ${ }^{a}$ Laboratoire de Techniques Aéronautiques et Spatiales, University of Liège, 4000 Liège, Belgium \\ ${ }^{\mathrm{b}}$ Department of Mechanical and Aerospace Engineering, Rutgers University, \\ Piscataway, NJ 08854, USA \\ ${ }^{\mathrm{c}}$ Graduate Aeronautical Laboratories, California Institute of Technology, Pasadena, CA 91125, USA
}

Received 28 March 2001; accepted 18 August 2001

\begin{abstract}
The present paper is concerned with the development of a micromechanical model of the hardening, rate-sensitivity and thermal softening of bcc crystals. In formulating the model, we specifically consider the following unit processes: double-kink formation and thermally activated motion of kinks; the close-range interactions between primary and forest dislocations, leading to the formation of jogs; the percolation motion of dislocations through a random array of forest dislocations introducing short-range obstacles of different strengths; dislocation multiplication due to breeding by double cross-slip; and dislocation pair annihilation. The model is found to capture salient features of the behavior of Ta crystals such as: the dependence of the initial yield point on temperature and strain rate; the presence of a marked stage I of easy glide, specially at low temperatures and high strain rates; the sharp onset of stage II hardening and its tendency to shift towards lower strains, and eventually disappear, as the temperature increases or the strain rate decreases; the parabolic stage II hardening at low strain rates or high temperatures; the stage II softening at high strain rates or low temperatures; the trend towards saturation at high strains; the temperature and strain-rate dependence of the saturation stress; and the orientation dependence of the hardening rate. (C) 2002 Published by Elsevier Science Ltd.
\end{abstract}

Keywords: A. Dislocations; B. Constitutive behavior; B. Crystal plasticity; B. Finite strain

\section{Introduction}

The present paper is concerned with the development of a micromechanical model of the hardening, rate-sensitivity and thermal softening of bcc crystals. We place

\footnotetext{
* Corresponding author. Fax: +1-626-449-6359.

E-mail address: ortiz@aero.caltech.edu (M. Ortiz).
} 
primary emphasis on the derivation of closed-form analytical expressions describing the macroscopic behavior of the crystals amenable to implementation as constitutive relations within a standard finite-element code. In developing the model, we follow the well-established paradigm of micromechanical modeling, consisting of the identification of the dominant or rate-limiting 'unit' processes operating at the microscale, the identification of the macroscopic forces driving the unit processes, the analysis of the response of the unit processes to the macroscopic driving forces, and the determination of the average or macroscopic effect of the combined operation of all the micromechanical unit processes (see, e.g., Bulatov and Kubin (1998); Phillips (1998); Campbell et al. (1998); Phillips et al. (1999); Moriarty et al. (1999); Baskes (1999) for recent overviews and discussions pertaining to micromechanics and multiscale modeling of materials).

In formulating the present model we specifically consider the following unit processes: double-kink formation and thermally activated motion of kinks; the close-range interactions between primary and forest dislocations, and the subsequent formation of jogs; the percolation motion of dislocations through a random array of forest dislocations introducing short-range obstacles of different strengths; dislocation multiplication due to breeding by double cross-slip; and dislocation pair annihilation. We believe that this forms an 'irreducible' set of unit processes, in that each of these processes accounts for-and is needed for matching - salient and clearly recognizable features of the experimental record. For instance, consideration of dislocation multiplication and annihilation leads to a predicted stage I-II transition strain which decreases with temperature and increases with strain rate, as observed experimentally.

We bring an assortment of analysis tools to bear on each of the unit processes under consideration. As already mentioned, the choice of tools is to a large extent conditioned by our desire to derive closed-form analytical expressions for all constitutive relations. The motion of dislocations through an otherwise defect-free lattice is assumed to be thermally activated and controlled by the nucleation of kink pairs. A analysis of this process based on transition-state theory yields the effective Peierls stress of the lattice as a function of temperature and strain rate. The short-range interactions between primary and secondary dislocations are assumed to result in the acquisition by both dislocations of a jog. The process by which the primary dislocation unpins from a forest obstacle is assumed to be thermally activated and the activation energy barrier is identified with the jog-formation energy. These assumptions result in temperature and rate-dependent obstacle strengths which are in good qualitative and quantitative agreement with experiment.

The percolation motion of dislocations through random arrays of point obstacles is studied in detail using statistical methods. The analysis presented here generalizes the analysis of Cuitiño and Ortiz (1992) so as to account for multi-species distributions of finite-strength obstacles. Finally, we model dislocation multiplication as the result of two competing effects: the proliferation of dynamic sources by double cross-slip; and dislocation pair annihilation. We model cross-slip as a thermally activated process and develop an elastic model, similar to that of Huang et al. (1999), which estimates the probability that the trajectories of two parallel screw dislocations collide, resulting in the annihilation of the pair. The model predicts that 
multiplication and annihilation balance out at sufficiently large strains, leading to saturation.

We validate the micromechanical model by recourse to detailed comparisons with the uniaxial tension tests on Ta single crystals of Mitchell and Spitzig (1965). The model is found to capture salient features of the behavior of Ta crystals such as the dependence of the initial yield point on temperature and strain rate, the presence of a marked stage I of easy glide, specially at low temperature and high strain rates, the sharp onset of stage II hardening and its tendency to shift towards lower strains, and eventually disappear, as the temperature increases or the strain rate decreases, the parabolic stage II hardening at low strain rates or high temperatures, the stage II softening at high strain rates or low temperatures, the trend towards saturation at high strains, the temperature and strain-rate dependence of the saturation stress, and the orientation dependence of the hardening rate.

\section{General framework}

Our objective is to formulate a model of the hardening of bcc crystals which is well-suited to finite-deformation large-scale finite-element calculations and, therefore, we couch the model within a non-linear kinematics framework. To this end, we adopt a conventional multiplicative elastic-plastic kinematics of the form (Lee, 1969; Teodosiu, 1969; Asaro and Rice, 1977; Havner, 1973; Hill and Rice, 1972; Rice, 1971):

$$
\boldsymbol{F}=\boldsymbol{F}^{\mathrm{e}} \boldsymbol{F}^{\mathrm{p}},
$$

where $\boldsymbol{F}$ is the deformation gradient, $\boldsymbol{F}^{\mathrm{e}}$ is the elastic lattice distortion and rotation, and $\boldsymbol{F}^{\mathrm{p}}$ is the plastic deformation, which represents the net effect of crystallographic slip and leaves the lattice undistorted and unrotated. In addition, the plastic deformation is volume preserving. In materials such as metals, the elastic response is ostensibly independent of the internal processes and the free energy density decomposes additively as

$$
A=W^{\mathrm{e}}\left(\boldsymbol{F}^{\mathrm{e}}, T\right)+W^{\mathrm{p}}\left(T, \boldsymbol{F}^{\mathrm{p}}, \boldsymbol{Q}\right),
$$

where $T$ is the absolute temperature and $\boldsymbol{Q}$ is some suitable set of internal variables. The function $W^{\mathrm{e}}$ determines the elastic response of the metal, e.g., upon unloading, whereas the function $W^{\mathrm{p}}$ describes the hardening of the crystal. Physically, $W^{\mathrm{p}}$ measures the energy stored in the crystal due to the plastic working of the material.

The examples of validation reported subsequently probe the hardening behavior of the material due to crystallographic slip over a range of moderate temperatures and strain rates. We therefore eschew a number of issues which become important at high pressures and elevated temperatures, but which play a limited role otherwise, including the volumetric equation of state (EoS), the pressure dependence of yield and elastic moduli, and others. First-principles calculations of the EoS and elastic moduli of bcc metals up to high pressures and temperatures may be found in Wasserman et al. (1996); Cohen et al. (1997, 2000), Soderlind and Moriarty (1998), Steinle-Neumann et al. (1999), Bulatov et al. (1999). These results may be used as a basis for extending 
the present theory to conditions such as arise in shocked materials. These extensions notwithstanding, a simple form of the elastic energy density appropriate for present purposes is

$$
W^{\mathrm{e}}\left(\boldsymbol{F}^{\mathrm{e}}, T\right)=\frac{1}{2} c_{i j k l}(T)\left(\varepsilon_{i j}^{\mathrm{e}}-\alpha_{i j} T\right)\left(\varepsilon_{k l}^{\mathrm{e}}-\alpha_{k l} T\right),
$$

where $c$ is the tensor of elastic moduli,

$$
\varepsilon^{\mathrm{e}}=\log \sqrt{\boldsymbol{C}^{\mathrm{e}}}=\frac{1}{2} \log \left(\boldsymbol{F}^{\mathrm{e} T} \boldsymbol{F}^{\mathrm{e}}\right)
$$

is the logarithmic elastic strain, and $\alpha$ is the thermal expansion tensor. The elastic properties of a cubic crystal are fully described by the three Voigt constants $c_{11}, c_{12}$ and $c_{44}$ and a scalar thermal expansion coefficient $\alpha$. For low temperatures, the temperature dependence of the moduli may be taken to be linear in $T$ to a first approximation, leading to the relation:

$$
c_{i j k l}(T) \approx c_{i j k l}^{0}-T c_{i j k l}^{1} .
$$

This linear dependence of the elastic moduli is observed experimentally up to moderate temperatures (Simmons and Wang, 1971) and can be justified within the framework of statistical mechanics (Weiner, 1983).

Plastic deformations in single crystals are crystallographic in nature. Following Rice (1971), we adopt a flow rule of the form

$$
\dot{\boldsymbol{F}}^{\mathrm{p}}=\left(\sum_{\alpha=1}^{N} \dot{\gamma}^{\alpha} \boldsymbol{s}^{\alpha} \otimes \boldsymbol{m}^{\alpha}\right) \boldsymbol{F}^{\mathrm{p}},
$$

where $\gamma^{\alpha}$ is the slip strain, and $\boldsymbol{s}^{\alpha}$, and $\boldsymbol{m}^{\alpha}$ are orthogonal unit vectors defining the slip direction and slip-plane normal corresponding to slip system $\alpha$. The collection $\gamma$ of slip strains may be regarded as a subset of the internal variable set $\boldsymbol{Q}$. A zero value of a slip rate $\dot{\gamma}^{\alpha}$ signifies that the corresponding slip system $\alpha$ is inactive. The flow rule (6) allows for multiple slip, i.e., for simultaneous activity on more than one system over a region of the crystal. The vectors $\left\{\boldsymbol{s}^{\alpha}, \boldsymbol{m}^{\alpha}\right\}$ remain constant throughout the deformation and are determined by crystallography. For bcc crystals, we consider the 24 slip systems listed in Table 1 in the Schmid and Boas nomenclature.

Slip on the $\left\{\begin{array}{lll}1 & 12\end{array}\right\}$ systems is known to be asymmetric at low temperatures: slip is easier when the applied stress is such that dislocations move in the twinning direction (e.g., Hull and Bacon (1984); Bengus et al. (1985); Seeger and Hollang (2000)). However, over the temperature range of interest here, the experimental evidence (Seeger and Hollang, 2000; Franciosi, 1983) suggests that the extent of the twinning/anti-twinning asymmetry is relatively small, e.g., of the order of $20 \mathrm{MPa}$ for Mo at $150 \mathrm{~K}$ (Seeger and Hollang, 2000) and, for simplicity, we will neglect it for a first approximation.

In the examples presented in Section 7, the constitutive equations are integrated in time using the variational update of Ortiz and Stainier (1999). The variational formulation of the rate problem proposed by Ortiz and Stainier (1999) additionally furnishes a convenient avenue for the superposition of the various unit mechanisms analyzed subsequently. Our working assumption is that the dissipation rates arising from these mechanisms are additive. This assumption in turn implies that the critical resolved shear 
Table 1

Slip systems of bcc crystals

\begin{tabular}{|c|c|c|c|c|c|c|}
\hline Syst. & Direction & Plane & Syst. & Direction & Plane & Group \\
\hline$A 2$ & {$\left[\begin{array}{lll}\overline{1} & 1 & 1\end{array}\right]$} & $(0 \overline{1} 1)$ & $A 2^{\prime}$ & {$\left[\begin{array}{lll}\overline{1} & 1 & 1\end{array}\right]$} & $\left(\begin{array}{llll}2 & 1 & 1\end{array}\right)$ & A \\
\hline$A 3$ & {$\left[\begin{array}{lll}1 & 1 & 1\end{array}\right]$} & $\left(\begin{array}{lll}1 & 0 & 1\end{array}\right)$ & $A 3^{\prime}$ & {$\left[\begin{array}{lll}1 & 1 & 1\end{array}\right]$} & $(12 \overline{1})$ & $\mathrm{T}$ \\
\hline$A 6$ & {$\left[\begin{array}{lll}\overline{1} & 1 & 1\end{array}\right]$} & $\left(\begin{array}{lll}1 & 1 & 0\end{array}\right)$ & $A 6^{\prime}$ & {$\left[\begin{array}{lll}\overline{1} & 1 & 1\end{array}\right]$} & 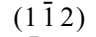 & $\mathrm{T}$ \\
\hline$B 2$ & {$\left[\begin{array}{lll}1 & 1 & 1\end{array}\right]$} & $\left(\begin{array}{lll}0 & \overline{1} & 1\end{array}\right)$ & $B 2^{\prime \prime}$ & {$\left[\begin{array}{lll}1 & 1 & 1\end{array}\right]$} & $(\overline{2} 11)$ & A \\
\hline$B 4$ & {$\left[\begin{array}{lll}1 & 1 & 1\end{array}\right]$} & $\left(\begin{array}{lll}1 & 0 & 1\end{array}\right)$ & $B 4^{\prime}$ & {$\left[\begin{array}{lll}1 & 1 & 1\end{array}\right]$} & $(1 \overline{2} 1)$ & A \\
\hline$B 5$ & {$\left[\begin{array}{lll}1 & 1 & 1\end{array}\right]$} & $\left(\begin{array}{lll}1 & 1 & 0\end{array}\right)$ & $B 5^{\prime}$ & {$\left[\begin{array}{lll}1 & 1 & 1\end{array}\right]$} & $\left(\begin{array}{lll}1 & 1 & \overline{2}\end{array}\right)$ & A \\
\hline$C 1$ & {$\left[\begin{array}{lll}1 & 1 & \overline{1}\end{array}\right]$} & $\left(\begin{array}{lll}0 & \overline{1} & 1\end{array}\right)$ & $C 1^{\prime}$ & {$\left[\begin{array}{lll}1 & 1 & 1\end{array}\right]$} & $(2 \overline{1} 1)$ & $\mathrm{T}$ \\
\hline$C 3$ & {$\left[\begin{array}{lll}1 & 1 & 1\end{array}\right]$} & $\left(\begin{array}{lll}1 & 0 & 1\end{array}\right)$ & $\mathrm{C}^{\prime \prime}$ & {$\left[\begin{array}{lll}1 & 1 & 1\end{array}\right]$} & (1) 21$)$ & $\mathrm{T}$ \\
\hline$C 5$ & {$\left[\begin{array}{lll}1 & 1 & 1\end{array}\right]$} & $\left(\begin{array}{lll}1 & 1 & 0\end{array}\right)$ & $C 5^{\prime \prime}$ & {$\left[\begin{array}{lll}1 & 1 & 1\end{array}\right]$} & $\left(\begin{array}{lll}1 & 1 & 2\end{array}\right)$ & A \\
\hline$D 1$ & {$\left[\begin{array}{lll}1 & 1 & 1\end{array}\right]$} & $(0 \overline{1} 1)$ & $D 1^{\prime \prime}$ & {$\left[\begin{array}{lll}1 & 1 & 1\end{array}\right]$} & $\left(\begin{array}{lll}2 & 1 & \overline{1}\end{array}\right)$ & $\mathrm{T}$ \\
\hline$D 4$ & {$\left[\begin{array}{lll}1 & 1 & 1\end{array}\right]$} & $\left(\begin{array}{lll}1 & 0 & 1\end{array}\right)$ & $D 4^{\prime \prime}$ & {$\left[\begin{array}{lll}1 & 1 & 1\end{array}\right]$} & (1 21$)$ & A \\
\hline$D 6$ & {$\left[\begin{array}{lll}1 & 1 & 1\end{array}\right]$} & $\left(\begin{array}{lll}1 & 1 & 0\end{array}\right)$ & $D 6^{\prime \prime}$ & {$\left[\begin{array}{lll}1 & 1 & 1\end{array}\right]$} & 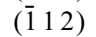 & $\mathrm{T}$ \\
\hline
\end{tabular}

stress $\tau_{\mathrm{c}}^{\alpha}$ for the operation of system $\alpha$ may be computed as the sum of unit-process contributions, i.e.,

$$
\tau_{\mathrm{c}}^{\alpha}=\sum_{\text {processes }} \tau_{\mathrm{c}}^{\alpha}(\text { process })
$$

where the sum extends to all unit processes. In the present model, these contributions are the Peierls stress of the lattice and the forest-obstacle resistance. Other dissipation mechanisms, such as phonon drag, may in principle be superposed likewise.

\section{Dislocation mobility}

In this section, we begin by considering the thermally activated motion of dislocations within an obstacle-free slip plane. Under these conditions, the motion of the dislocations is driven by an applied resolved shear stress $\tau$ and is hindered by the lattice resistance, which is weak enough that it may be overcome by thermal activation. The lattice resistance is presumed to be well-described by a Peierls energy function, which assigns an energy per unit length to dislocation segments as a function of their position on the slip plane.

In bcc crystals, the core of screw dislocation segments relaxes into low-energy non-planar configurations (Duesbery et al., 1973; Vitek, 1976, 1992; Xu and Moriarty, 1996; Duesbery and Vitek, 1998; Moriarty et al., 1999; Ismail-Beigi and Arias, 2000; Wang et al., 2000). This introduces deep valleys into the Peierls energy function aligned with the Burgers vector directions and possessing the periodicity of the lattice. At low temperatures, the dislocations tend to adopt low-energy configurations and, consequently, the dislocation population predominantly consists of long screw segments. In order to move a screw segment normal to itself, the dislocation core must first be constricted, which requires a substantial supply of energy. Thus, the energy barrier for the motion of screw segments, and the attendant Peierls stress, may be expected to be large, and the energy barrier for the motion of edge segments to be comparatively 


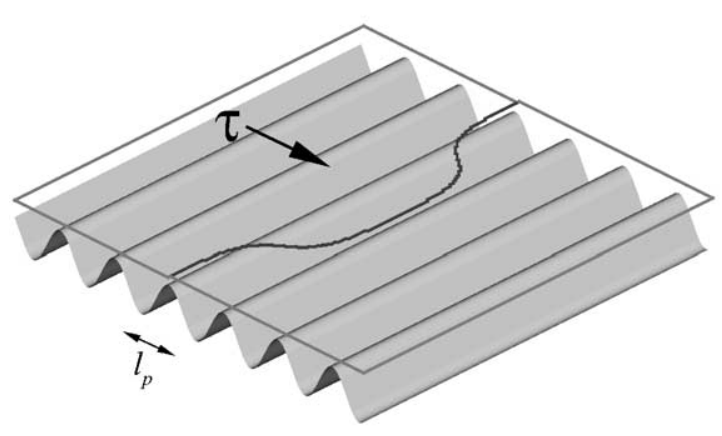

(a)

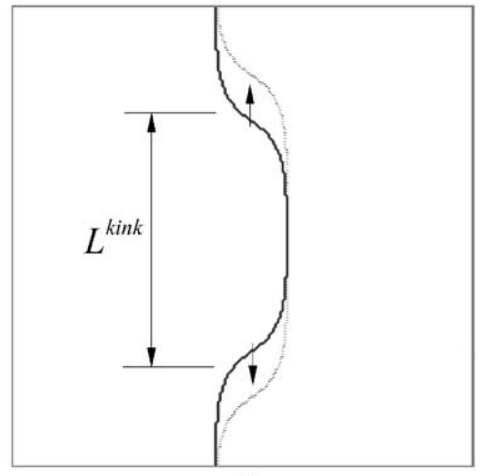

(b)

Fig. 1. Schematic of the double-kink mechanism.

smaller. For instance, Duesbery and Xu (1998) have calculated the Peierls stress for a rigid screw dislocation in Mo to be $0.022 \mu$, where $\mu$ is the $\langle 111\rangle$ shear modulus, whereas the corresponding Peierls stress for a rigid edge dislocation is $0.006 \mu$, or about one-fourth of the screw value. This suggests that the rate-limiting mechanism for dislocation motion is the thermally activated motion of kinks along screw segments (Hirsch, 1960; Seeger and Schiller, 1962; Hirth and Lothe, 1968).

Consider now a screw dislocation segment, possibly pinned at both ends by an obstacle pair, subjected to a resolved shear stress $\tau>0$. At sufficiently high temperatures a double-kink may be nucleated with the assistance of thermal activation (e.g., Hirth and Hoagland (1993); Xu and Moriarty (1998); Moriarty et al. (1999)), and the subsequent motion of the kinks causes the screw segment to effectively move forward (Fig. 1). Neglecting entropic effects and invoking Peach-Koehler's formula, the forward and backward activation enthalpies $\Delta G^{+}$and $\Delta G^{-}$, respectively, for the formation of a double kink may be approximated as

$$
\Delta G^{ \pm} \approx E^{\mathrm{kink}} \mp b \tau L^{\mathrm{kink}} l_{\mathrm{P}}
$$

where $E^{\mathrm{kink}}$ is the energy of formation of a kink-pair, $L^{\mathrm{kink}}$ is the length of an incipient double kink, and $l_{\mathrm{P}}$ is the distance between two consecutive Peierls valleys. For bcc crystals, $l_{\mathrm{P}}=\sqrt{2 / 3} a$ if the slip plane is $\left\{\begin{array}{lll}1 & 10 & 0\end{array}, l_{\mathrm{P}}=\sqrt{2} a\right.$, if the slip plane is $\left\{\begin{array}{lll}1 & 12 & 2\end{array}\right.$, and $l_{\mathrm{P}}=\sqrt{8 / 3} a$ if the slip plane is $\{123\}$, where $a$ is the cubic lattice size (Seeger and Hollang, 2000).

The formation energy $E^{\text {kink }}$ cannot be reliably estimated from elasticity since it is composed mostly of core region. It can, however, be accurately computed by recourse to atomistic models. For instance, for Mo at zero stress $\mathrm{Xu}$ and Moriarty (1998) have found formation energies $E^{\text {kink }}$ of the order of $1 \mathrm{eV}$ for kinks separated by a distance greater than $L^{\text {kink }}=15 b$. The core structure, gamma surfaces, Peierls stress, and kink-pair formation energies associated with the motion of $a / 2\langle 111\rangle$ screw dislocations in $\mathrm{Ta}$ and Mo have also been calculated by Moriarty et al. (1999). 
We may expect a proliferation of kinks at a stress of the order of

$$
\tau_{0}=\frac{E^{\mathrm{kink}}}{b L^{\mathrm{kink}} l_{\mathrm{P}}}
$$

for which $\Delta G=0$. For Mo, Xu and Moriarty (1998) have computed $\tau_{0}$ to be of the order of a few GPa. The activation free enthalpy for double kink nucleation may thus be rewritten as

$$
\Delta G^{ \pm}(\tau)=E^{\mathrm{kink}}\left(1 \pm \frac{\tau}{\tau_{0}}\right), \quad 0 \leqslant \tau<\tau_{0} .
$$

A slightly more elaborate empirical formula that is widely used to fit activation energies was proposed by Kocks et al. (1975) and the corresponding parameters have been determined for Ta by Tang et al. (1998), but this enhancement will not be pursued here.

Transition state theory predicts that a dislocation segment effects $v_{\mathrm{D}} \mathrm{e}^{-\beta G^{+}}$and $v_{\mathrm{D}} \mathrm{e}^{-\beta G^{-}}$jumps per unit time in the positive and negative directions, respectively. Here, $\beta=1 / k_{\mathrm{B}} T, k_{\mathrm{B}}$ is Boltzmann's constant, $T$ is the absolute temperature, and $v_{\mathrm{D}}$ is the attempt frequency which may be identified with the Debye frequency to a first approximation. Since the length of the individual jumps is $l_{\mathrm{P}}$, the mean velocity of the dislocations then follows as

$$
v=2 l_{\mathrm{P}} v_{\mathrm{D}} \mathrm{e}^{-\beta E^{\mathrm{kink}}} \sinh \left(\beta E^{\mathrm{kink}} \frac{\tau}{\tau_{0}}\right) .
$$

An application of Orowan's formula then gives the slip strain rate as

$$
\dot{\gamma}=\dot{\gamma}_{0}^{\text {kink }} \mathrm{e}^{-\beta E^{\mathrm{kink}}} \sinh \left(\beta E^{\mathrm{kink}} \frac{\tau}{\tau_{0}}\right),
$$

where

$$
\dot{\gamma}_{0}^{\text {kink }}=2 b \rho l_{\mathrm{P}} v_{\mathrm{D}}
$$

is a reference strain rate, and $\rho$ is the dislocation density. In writing Eq. (12), we have taken into account the possibility of thermally activated jumps in the reverse direction, with forward and backward activation enthalpies $\Delta G^{+}$and $\Delta G^{-}$, respectively. For slip in the positive direction, $\dot{\gamma} \geqslant 0$, Eq. (12) may be inverted to give

$$
\frac{\tau_{\mathrm{P}}}{\tau_{0}}=\frac{1}{\beta E^{\text {kink }}} \operatorname{asinh}\left(\frac{\dot{\gamma}}{\gamma_{0}^{\text {kink }}} \mathrm{e}^{\beta E^{\text {kink }}}\right),
$$

where $\tau_{\mathrm{P}}$ may be regarded as a temperature and rate-dependent effective Peierls stress.

Fig. 2 illustrates the dependence of the effective Peierls stress on temperature and rate of deformation. The Peierls stress decreases ostensibly linearly up to a critical temperature $T_{\mathrm{c}}$, beyond which it rapidly tends to zero. These trends are in agreement with the experimental observations of Wasserbäch (1986) and Lachenmann and Schultz (1970). The critical temperature $T_{\mathrm{c}}$ increases with the strain rate. In particular, in this model the effect of increasing (decreasing) the strain rate has an analogous effect to decreasing (increasing) the temperature, and vice-versa, as noted by Tang et al. (1999). In the regime of very high strain-rates $\left(\dot{\gamma}>10^{5} \mathrm{~s}^{-1}\right)$, effects such as electron and photon drag become important and control the velocity of dislocations (Suzuki et al., 1991; Brailsford, 1969). 


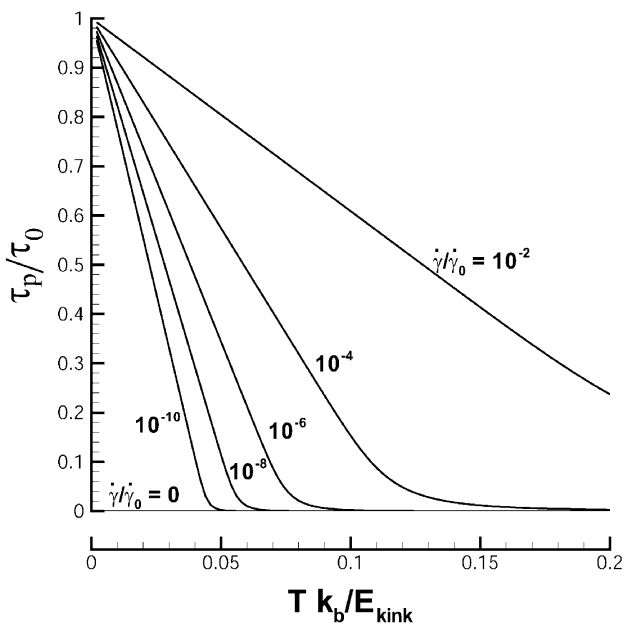

Fig. 2. Temperature dependence of the effective Peierls stress for various strain rates. Note that the typical order of magnitude of $\gamma_{0}^{\text {kink }}=10^{6} \mathrm{~s}^{-1}$.

\section{Forest hardening}

In the forest-dislocation theory of hardening, the motion of dislocations, which are the agents of plastic deformation in crystals, is impeded by secondary - or 'forest' dislocations crossing the slip plane. As the moving and forest dislocations intersect, they form jogs or junctions of varying strengths (Baskes et al., 1998; Rodney and Phillips, 1999; Phillips et al., 1999; Shenoy et al., 2000; Danna and Benoit, 1993; Rhee et al., 1998; Huang et al., 1999; Kubin et al., 1998; Zbib et al., 2000) which, provided the junction is sufficiently short, may be idealized as point obstacles. Moving dislocations are pinned down by the forest dislocations and require a certain elevation of the applied resolved shear stress in order to bow out and bypass the pinning obstacles. The net effect of this mechanism is macroscopic hardening. Detailed numerical simulations of a dislocation line propagating through forest dislocations have been carried out by Foreman and Makin (1966, 1967), and by Kocks (1966), and more recently by Tang et al. (1999). Analytical treatments of the model have been given by Kocks (1966), Ortiz and Popov (1982), and Cuitiño and Ortiz (1992) for the case of infinitely strong obstacles. A phase-field model of the forest hardening mechanism has been proposed by Ortiz (1999) and by Koslowski et al. (2001). Here, we extend the statistical analysis of Cuitiño and Ortiz (1992) to the case of several species of obstacles of finite strength.

Due to the random nature of the interactions, the motion of dislocations through a distribution of obstacles is best described in statistical terms (Ortiz and Popov, 1982). We begin by treating the case of infinitely strong obstacles. In this case, pairs of obstacles pin down dislocation segments, which require a certain threshold resolved shear stress $s$ in order to overcome the obstacle pair. Since the distribution of point obstacles within the slip plane is random, it follows that $s$ is itself a random variable. We shall let $\tilde{f}_{0}^{\alpha}(s, t)$ denote the probability density function of two-point barrier strengths on slip 


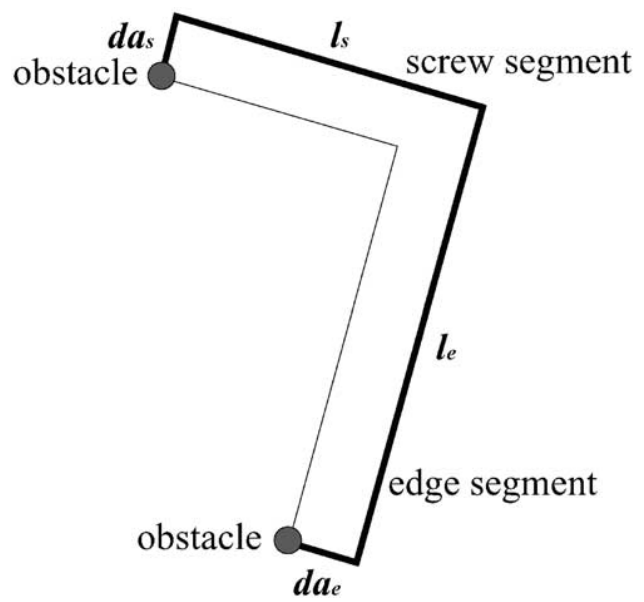

Fig. 3. Bow-out mechanism for a dislocation segment bypassing an obstacle pair.

system $\alpha$ at time $t$. The time dependence of $\tilde{f}_{0}^{\alpha}(s, t)$ is a consequence of the variation in forest dislocation density.

In order to determine the precise form of $\tilde{f}_{0}^{\alpha}(s, t)$, we begin by noting that the Peierls energy landscape of bcc crystals strongly favors either screw or edge segments (Chang et al., 1999). In addition kinks, or points of change of direction of the dislocation line, carry a non-negligible amount of energy. It therefore follows that the lowest-energy configuration of unstressed dislocation segments spanning an obstacle pair is a step of the form shown in Fig. 3. Under these conditions, the bow-out mechanism by which a dislocation segment bypasses an obstacle pair may be expected to result in the configuration shown in Fig. 3 (bold line). If the edge-segment length is $l_{\mathrm{e}}$, a displacement $\mathrm{da}_{\mathrm{e}}$ of the dislocation requires a supply of energy equal to $2 U^{\mathrm{screw}} \mathrm{da}_{\mathrm{e}}+$ $b \tau_{\mathrm{P}}^{\text {edge }} l_{\mathrm{e}} \mathrm{da}_{\mathrm{e}}$ in order to overcome the Peierls resistance $\tau_{\mathrm{P}}^{\text {edge }}$ and to extend the screw segments. The corresponding energy release is $b \tau l_{\mathrm{e}} \mathrm{da}_{\mathrm{e}}$. Similar contributions result from a displacement $\mathrm{da}_{\mathrm{s}}$ of the screw-segment of length $l_{\mathrm{s}}$. Therefore, for the bow-out of the dislocation to be energetically possible we must have

$$
b \tau l_{\mathrm{e}} \mathrm{da}_{\mathrm{e}}+b \tau l_{\mathrm{s}} \mathrm{da}_{\mathrm{s}} \geqslant 2 U^{\text {screw }} \mathrm{da}_{\mathrm{e}}+b \tau_{\mathrm{P}}^{\text {edge }} l_{\mathrm{e}} \mathrm{da}_{\mathrm{e}}+2 U^{\text {edge }} \mathrm{da}_{\mathrm{s}}+b \tau_{\mathrm{P}}^{\text {screw }} l_{\mathrm{s}} \mathrm{da}_{\mathrm{s}} .
$$

As already noted, in bcc crystals the core energy $U^{\text {screw }}$ per unit length of the screw segments is smaller than the core energy $U^{\text {edge }}$ per unit length of the edge segments (e.g., Wang et al. (2000)). Conversely, the Peierls resistance $\tau_{\mathrm{P}}^{\text {screw }}$ to the glide of screw segments is larger than the Peierls resistance $\tau_{\mathrm{P}}^{\text {edge }}$ for edge segments (e.g., Duesbery and $\mathrm{Xu}$ (1998)). Retaining dominant terms only, Eq. (15) simplifies to

$$
\tau \geqslant s=\tau_{\mathrm{P}}^{\text {screw }}+\frac{2 U^{\text {edge }}}{b l_{\mathrm{s}}} .
$$

We may further identify the Peierls stress $\tau_{\mathrm{P}}^{\text {screw }}$ with Eq. (14), whence it follows that $s$ comprises a thermally activated and rate-sensitive term $\tau_{\mathrm{P}}$ and an athermal and rate-insensitive term $2 U^{\text {edge }} / b l_{\mathrm{s}}$. 
We shall assume that the point obstacles are randomly distributed over the slip plane with a mean density $n^{\alpha}$ of obstacles per unit area. We shall also assume that the obstacle pairs spanned by dislocation segments are nearest-neighbors in the obstacle ensemble. Thus, if $r$ is the distance between the obstacles in an obstacle pair, then the circle of radius $r$ centered at either obstacle contains no other obstacles. Under these conditions, the probability density of $r$ is given by (Kocks 1966; Cuitiño and Ortiz 1992)

$$
\tilde{f}_{0}^{\alpha}(r, t)=2 \pi n^{\alpha} r \exp \left(-\pi n^{\alpha} r^{2}\right)
$$

where the dependence of $\tilde{f}_{0}$ on time stems from the time dependence of the obstacle density $n^{\alpha}$.

In order to deduce the probability density of $l_{\mathrm{s}}$, and by extension of $s$, we note that, given a point obstacle located at the origin, the probability of finding another obstacle in the element of area $r \mathrm{~d} r \mathrm{~d} \theta$ is

$$
\mathrm{d} \tilde{P}_{0}^{\alpha}(r, t)=n^{\alpha} r \exp \left(-\pi n^{\alpha} r^{2}\right) \mathrm{d} r \mathrm{~d} \theta
$$

Changing variables to Cartesian coordinates, $x=r \cos \theta, y=r \sin \theta$, Eq. (18) may be recast in the form

$$
\mathrm{d} \tilde{P}_{0}^{\alpha}=n^{\alpha} \exp \left[-\pi n^{\alpha}\left(x^{2}+y^{2}\right)\right] \mathrm{d} x \mathrm{~d} y .
$$

The frequency of obstacle pairs with screw-segment length $l_{\mathrm{s}}$ is, therefore

$$
\tilde{f}_{0}^{\alpha}\left(l_{\mathrm{s}}, t\right)=2 \int_{-\infty}^{\infty} n^{\alpha} \exp \left[-\pi n^{\alpha}\left(x^{2}+l_{\mathrm{s}}^{2}\right)\right] \mathrm{d} x=\sqrt{n^{\alpha}} \exp \left(-\pi n^{\alpha} l_{\mathrm{s}}^{2}\right) .
$$

The corresponding probability of finding an obstacle pair of strength $s$ follows from Eqs. (20) and (16) as

$$
\tilde{f}_{0}^{\alpha}(s, t)=\frac{4 U^{\text {edge }} \sqrt{n^{\alpha}}}{b\left(s-\tau_{\mathrm{P}}^{\alpha}\right)^{2}} \exp \left[-\frac{\pi n^{\alpha}}{b^{2}}\left(\frac{2 U^{\text {edge }}}{s-\tau_{\mathrm{P}}^{\alpha}}\right)^{2}\right], \quad s \geqslant \tau_{\mathrm{P}}^{\alpha}
$$

and the associated distribution function is

$$
\tilde{P}_{0}^{\alpha}(s, t)=\int_{\tau_{\mathrm{P}}}^{s} \tilde{f}_{0}^{\alpha}(\xi, t) \mathrm{d} \xi=\left[1-\operatorname{erf}\left(\frac{2 \sqrt{\pi n^{\alpha}} U^{\text {edge }}}{b\left(s-\tau_{\mathrm{P}}^{\alpha}\right)}\right)\right] .
$$

We note that the Peierls stress $\tau_{\mathrm{P}}^{\alpha}$ depends on the slip system $\alpha$ through its dependence on the slip-strain rate $\dot{\gamma}^{\alpha}$.

It is interesting to note that the probability density $\tilde{f}_{0}^{\alpha}(s, t)$ of obstacle-pair strengths just derived for bcc crystals differs markedly from those which are obtained for fcc crystals by a similar argument (Kocks, 1966; Mughrabi, 1975; Grosskreutz and Mughrabi, 1975; Cuitiño and Ortiz, 1992), namely

$$
\tilde{f}_{0}^{\alpha}(s, t)=\frac{2 \pi n^{\alpha} U^{2}}{b^{2} s^{3}} \exp \left(-\frac{n^{\alpha} \pi}{b^{2}} \frac{U^{2}}{s^{2}}\right),
$$

where $U$ is the dislocation core energy per unit length of dislocation. This difference owes to the different bow-out configurations for the two crystal classes and the comparatively larger values of the Peierls stress in bcc crystals. Thus, the Peierls stress 
of fcc crystals is generally quite small and, as in the derivation of Eq. (23) is often neglected entirely to a first approximation. For bcc crystals, the effective Peierls stress $\tau_{\mathrm{P}}^{\alpha}$ decreases with increasing temperature and decreasing rate of deformation, Eq. (14), and hence the behavior of bcc crystals may be expected to be closer to that of fcc crystals under those conditions, as noted by Tang et al. (1999). Conversely, the hardening behavior of bcc crystals may be expected to differ sharply from that of fcc crystals at low temperatures and high rates of deformation.

The function $\tilde{f}_{0}^{\alpha}(s, t)$ just derived provides a complete description of the distribution of the obstacle-pair strengths when the point obstacles are of infinite strength and, consequently, impenetrable to the dislocations. Next, we extend the preceding analysis to the case of finite obstacle strengths. Let $s^{\alpha \beta}$ be the strength of the jogs or junctions formed by dislocations of systems $\alpha$ and $\beta$. Now consider an obstacle pair in which the weakest point obstacle corresponds to a forest dislocation of type $\beta$ and, therefore, has strength $s^{\alpha \beta}$. The probability that the strength of the obstacle pair be $s$ is, therefore,

$$
\tilde{f}^{\alpha}\left(s \mid s^{\alpha \beta}, t\right)=\frac{\tilde{f}_{0}^{\alpha}(s, t)}{\tilde{P}_{0}^{\alpha}\left(s^{\alpha \beta}, t\right)}\left[1-H\left(s-s^{\alpha \beta}\right)\right],
$$

where $H(s)$ is the Heaviside function. The probability that an obstacle on system $\alpha$ be of type $\beta \neq \alpha$ is $n^{\alpha \beta} / n^{\alpha}$, where $n^{\alpha \beta}$ is the number of obstacles of type $\beta$ per unit area of the slip plane $\alpha$, and

$$
n^{\alpha}(t)=\sum_{\beta \neq \alpha} n^{\alpha \beta}(t)
$$

is the total obstacle density on slip system $\alpha$.

Next, we note that the probability that the weakest of the two obstacles forming a obstacle pair be of type $\beta$ is

$$
p^{\alpha \beta}=\frac{n^{\alpha \beta}}{n^{\alpha}}\left(\frac{n^{\alpha \beta}}{n^{\alpha}}+2 \sum_{s^{\alpha \beta^{\prime}}>s^{\alpha \beta}} \frac{n^{\alpha \beta^{\prime}}}{n^{\alpha}}\right), \quad \beta \neq \alpha, \quad \beta^{\prime} \neq \alpha .
$$

It is readily verified that

$$
\sum_{\beta \neq \alpha} p^{\alpha \beta}=\left[\sum_{\beta \neq \alpha}\left(\frac{n^{\alpha \beta}}{n^{\alpha}}\right)\right]^{2}=1
$$

as required. Finally, the probability that an obstacle pair have strength $s$ follows as

$$
\tilde{f}^{\alpha}(s, t)=\sum_{\beta \neq \alpha} p^{\alpha \beta}(t) \tilde{f}^{\alpha}\left(s \mid s^{\alpha \beta}, t\right) .
$$

This probability distribution function jointly accounts for the strength of the obstacle pairs due to line tension and to the obstacle strength. Evidently, $\tilde{f}^{\alpha}(s, t)$ is supported in the interval $\left[0, s_{\max }\right]$, where $s_{\max }$ is the maximum obstacle strength. Making use of 
Eq. (24), the probability density function (28) can be rewritten in the form

$$
\tilde{f}^{\alpha}(s, t)=\sum_{\beta \neq \alpha} p^{\alpha \beta}(t) \tilde{f}_{0}^{\alpha}(s, t) \frac{1-H\left(s-s^{\alpha \beta}\right)}{\tilde{P}_{0}^{\alpha}\left(s^{\alpha \beta}, t\right)}
$$

and the associated distribution function becomes

$$
\tilde{P}^{\alpha}(s, t)=\sum_{\beta \neq \alpha} p^{\alpha \beta}(t)\left\{\tilde{P}_{0}^{\alpha}(s, t) \frac{1-H\left(s-s^{\alpha \beta}\right)}{\tilde{P}_{0}^{\alpha}\left(s^{\alpha \beta}, t\right)}+H\left(s-s^{\alpha \beta}\right)\right\} .
$$

We assume that $n^{\alpha \beta}$, the number of obstacles of type $\beta$ per unit area of the slip plane $\alpha$, scales with the dislocation density $\rho^{\beta}$ according to the relation

$$
n^{\alpha \beta}=a^{\alpha \beta} \rho^{\beta},
$$

where the coupling constants $a^{\alpha \beta}$ are regarded here as purely geometrical parameters. A simple geometrical argument based on counting intersections of randomly distributed lines with a slip plane gives (Cuitiño and Ortiz, 1993)

$$
n^{\alpha \beta}=\frac{2}{\pi} \sqrt{1-\left(\boldsymbol{m}^{\alpha} \cdot \boldsymbol{m}^{\beta}\right)^{2}} \rho^{\beta},
$$

where $\boldsymbol{m}^{\alpha}$ and $\boldsymbol{m}^{\beta}$ are the unit normals to slip planes of types $\alpha$ and $\beta$ and $\rho^{\beta}$ is the dislocation line density per unit volume in slip system $\beta$. A comparison between Eqs. (31) and (32) suggests writing:

$$
a^{\alpha \beta}=a_{0} \frac{2}{\pi} \sqrt{1-\left(\boldsymbol{m}^{\alpha} \cdot \boldsymbol{m}^{\beta}\right)^{2}},
$$

where $a_{0}<1$ is an "efficiency" factor which accounts for the tendency of dislocations to tangle and form loops, which in turn tends to lower the number of slip-plane crossings.

Let $\tau^{\alpha}(t)$ now be the resolved shear stress acting on the slip system $\alpha$ at time $t$. Assume for now that $\tau^{\alpha}(t)$ increases monotonically from zero at $t=0$. Evidently, for dislocations to be stable at time $t$, they must face barriers of strengths $s$ in excess of $\tau^{\alpha}(t)$. As $\tau^{\alpha}(t)$ is increased to $\tau^{\alpha}(t)+\tau^{\alpha}(t) \mathrm{d} t$, the dislocation segments held at barriers of strengths in the range $\tau^{\alpha}(t) \leqslant s \leqslant \tau^{\alpha}(t)+\tau^{\alpha}(t) \mathrm{d} t$ are dislodged and move forward until they reach barriers of strength $s \geqslant \tau^{\alpha}(t)+\dot{\tau}^{\alpha}(t) \mathrm{d} t$. This motion of dislocations results in a net increase in the plastic deformation. The dislocation jumps between obstacles are assumed to be instantaneous. This idealization is justified when the duration of the flights is much smaller than the characteristic time of variation of the loads.

As noted by Ortiz and Popov (1982), the information needed to describe the dislocation motion is fully contained in the probability density function $f^{\alpha}(s, t)$, which represents the fraction of dislocation length facing obstacle pairs of strength $s$ at time $t$. The function $f^{\alpha}(s, t)$ evolves in time due to the process of redistribution of dislocation line described above. Initially, though, the dislocations may be assumed to be randomly distributed over their slip plane, and $f^{\alpha}(s, 0)=\tilde{f}^{\alpha}(s, 0)$. At later times, $f^{\alpha}(s, t)$ must vanish identically for $0 \leqslant s<\tau^{\alpha}(t)$ in the rate independent limit.

A kinetic equation for the evolution of $f^{\alpha}(s, t)$ was derived by Ortiz and Popov (1982) using standard tools of non-equilibrium statistical mechanics. Cuitiño and Ortiz 
(1992) were able to obtain an analytical solution to this kinetic equation for the case of monotonic loading and an arbitrary time variation of the density of point obstacles. Remarkably, the solution takes the simple closed form

$$
f^{\alpha}(s, t)=\frac{\tilde{f}^{\alpha}(s, t)}{1-\tilde{P}^{\alpha}\left(\tau^{\alpha}(t), t\right)} H\left(s-\tau^{\alpha}(t)\right) .
$$

Solution (34) implies that, under the conditions of the analysis, the probability density $f^{\alpha}(s, t)$ remains proportional to $\tilde{f}^{\alpha}(s, t)$ over the current admissible range $\left[\tau^{\alpha}(t), s_{\max }\right]$.

Let $\rho^{\alpha}(t)$ denote the current dislocation length per unit volume for the slip system $\alpha$. The dislocation density released during an increment of the resolved shear stress from $\tau^{\alpha}(t)$ to $\tau^{\alpha}(t)+i^{\alpha}(t) \mathrm{d} t$ gives rise to an incremental plastic strain (Kocks, 1966; Teodosiu, 1969)

$$
\left.\mathrm{d} \gamma^{\alpha}(t)=b \rho^{\alpha}(t) f\left(\tau^{\alpha}(t), t\right)\right) \dot{\tau}^{\alpha}(t) \mathrm{d} t \bar{N}(t) \bar{l}(t),
$$

where $\bar{l}(t)$ is the average distance between obstacles, and $\bar{N}(t)$ is the average number of jumps the dislocation segments make before attaining stable positions.

To a good approximation, $\bar{l}(t)$ can be identified with the average distance between point obstacles. Assuming that the obstacles are randomly distributed over the slip plane with density $n^{\alpha}(t)$, a straightforward derivation from Eq. (17) gives (Cuitiño and Ortiz, 1992)

$$
\bar{l}(t)=\langle l\rangle(t)=\frac{1}{2 \sqrt{n^{\alpha}(t)}} .
$$

Next, we compute the average number of jumps $\bar{N}(t)$. Evidently, the probability that an unstable segment becomes arrested after the first jump is equal to the probability that the first barrier encountered is of a strength $s \geqslant \tau^{\alpha}(t)$. This probability is $1-$ $\tilde{P}^{\alpha}\left(\tau^{\alpha}(t), t\right)$. The probability that the segment goes beyond the first barrier is $\tilde{P}^{\alpha}\left(\tau^{\alpha}(t), t\right)$. Likewise, the probability that a segment gets arrested at the second barrier encountered is $\tilde{P}^{\alpha}\left(\tau^{\alpha}(t), t\right)\left[1-\tilde{P}^{\alpha}\left(\tau^{\alpha}(t), t\right)\right]$, and the probability that it goes beyond is $\tilde{P}^{\alpha 2}\left(\tau^{\alpha}(t), t\right)$, and so on. Hence, the average number of jumps between barriers taken by an unstable segment is

$$
\begin{aligned}
\bar{N}(t)= & {\left[1-\tilde{P}^{\alpha}\left(\tau^{\alpha}(t), t\right)\right]+2 \tilde{P}^{\alpha}\left(\tau^{\alpha}(t), t\right)\left[1-\tilde{P}^{\alpha}\left(\tau^{\alpha}(t), t\right)\right] } \\
& +3 \tilde{P}^{\alpha 2}\left(\tau^{\alpha}(t), t\right)\left[1-\tilde{P}^{\alpha}\left(\tau^{\alpha}(t), t\right)\right]+\cdots=\frac{1}{1-\tilde{P}^{\alpha}\left(\tau^{\alpha}(t), t\right)} .
\end{aligned}
$$

Interestingly, if $\tau^{\alpha}=0$, any moving dislocation segment is sure to be arrested at the first obstacle it encounters, and $\bar{N}=1$, in agreement with Eq. (37). Likewise, if $\tau^{\alpha}(t)>s_{\max }$, then the segment never reaches a stable barrier and $\bar{N} \rightarrow \infty$, as predicted by Eq. (37).

Substituting Eqs. (37) and (34) with $s=\tau^{\alpha}(t)$ into Eq. (35) we finally obtain

$$
\dot{\gamma}^{\alpha}(t)=\gamma_{\mathrm{c}}^{\alpha}(t) \frac{\tilde{f}^{\alpha}\left(\tau^{\alpha}(t), t\right)}{\left[1-\tilde{P}^{\alpha}\left(\tau^{\alpha}(t), t\right)\right]^{2}} \dot{\tau}^{\alpha}(t),
$$


where the characteristic plastic strain $\gamma_{\mathrm{c}}^{\alpha}(t)$ is defined as

$$
\gamma_{c}^{\alpha}(t)=\frac{b \rho^{\alpha}(t)}{2 \sqrt{n^{\alpha}(t)}} .
$$

Eq. (38) defines a relation of the form

$$
\dot{\gamma}^{\alpha}(t)=\frac{\dot{\tau}^{\alpha}(t)}{h^{\alpha}(t)}
$$

where

$$
h^{\alpha}(t)=\frac{1}{\gamma_{c}^{\alpha}(t)} \frac{\left[1-\tilde{P}^{\alpha}\left(\tau^{\alpha}(t), t\right)\right]^{2}}{\tilde{f}^{\alpha}\left(\tau^{\alpha}(t), t\right)}
$$

is the hardening modulus of the slip system $\alpha$. By way of example, in the particular case of obstacles of uniform strength, $s^{\alpha \beta}=s_{\max }$, the hardening modulus takes the form

$$
\begin{aligned}
h^{\alpha}(t)= & h_{\mathrm{c}}^{\alpha}(t)\left(\frac{\tau^{\alpha}(t)-\tau_{\mathrm{P}}^{\alpha}}{\tau_{\mathrm{c}}^{\alpha}}\right)^{2} \frac{1}{\sqrt{\pi}} \exp \left[\left(\frac{\tau_{\mathrm{c}}^{\alpha}}{\tau^{\alpha}(t)-\tau_{\mathrm{P}}^{\alpha}}\right)^{2}\right] \\
& \times \frac{\left[\tilde{P}_{0}^{\alpha}\left(s_{\max }, t\right)-\tilde{P}_{0}^{\alpha}\left(\tau^{\alpha}, t\right)\right]^{2}}{\tilde{P}_{0}^{\alpha}\left(s_{\max }, t\right)}\left(1-H\left(\tau^{\alpha}-s_{\max }\right)\right),
\end{aligned}
$$

where

$$
\tau_{\mathrm{c}}^{\alpha}(t)=2 \sqrt{\pi n^{\alpha}} \frac{U^{\text {edge }}}{b}, \quad h_{\mathrm{c}}^{\alpha}(t)=\frac{\tau_{\mathrm{c}}^{\alpha}(t)}{\gamma_{\mathrm{c}}^{\alpha}(t)}
$$

are a characteristic shear stress and plastic modulus, respectively. Eq. (42) predicts an initial infinite hardening modulus at $\tau=\tau_{\mathrm{P}}$. The hardening modulus subsequently decreases monotonically to zero as $\tau$ approaches $s_{\max }$.

As shown in Cuitiño and Ortiz (1992), the above relations can be extended simply to account for elastic unloading, provided that $\tau^{\alpha}(t)$ does not change sign an time during the loading history. This extension consists in defining the current flow stress $g^{\alpha}(t)$ of slip system $\alpha$ as the maximum previously attained value of $\tau^{\alpha}(t)$. The hardening relations are then rewritten in the form

$$
\dot{\gamma}^{\alpha}(t)=\frac{\dot{g}^{\alpha}(t)}{h^{\alpha}(t)}
$$

and

$$
h^{\alpha}(t)=\frac{1}{\gamma_{c}^{\alpha}(t)} \frac{\left[1-\tilde{P}^{\alpha}\left(g^{\alpha}(t), t\right)\right]^{2}}{\tilde{f}^{\alpha}\left(g^{\alpha}(t), t\right)} .
$$

It should be noted that, since $\dot{g}^{\alpha}(t)=0$ whenever either $\tau^{\alpha}(t)<g^{\alpha}(t)$ or $\dot{\tau}^{\alpha}(t) \leqslant 0$, relation (44) implicitly accounts for elastic unloading. To account for the initial distribution of dislocations and obstacles, we take $g^{\alpha}(t)=g_{0}$.

\section{Dislocation intersections}

In this section, we proceed to estimate the obstacle strengths which enter in the preceding analysis of forest hardening. The interaction between primary and secondary 


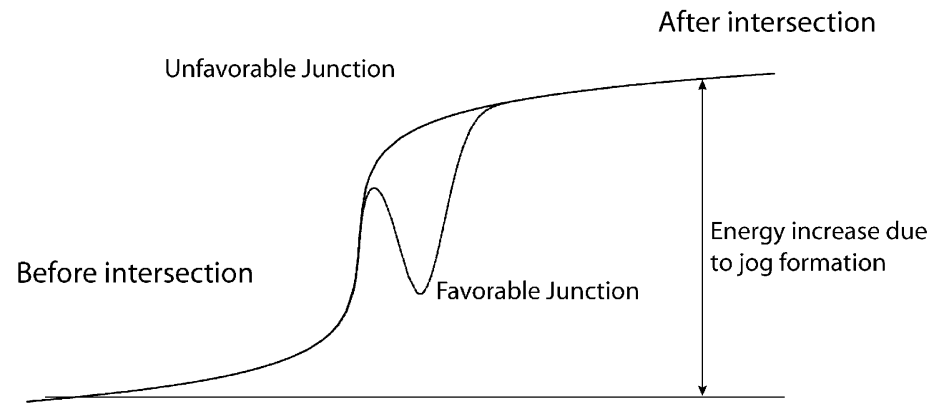

Fig. 4. Schematic of energy variation as a function of a reaction coordinate during dislocation intersection and crossing.

dislocations may result in a variety of reaction products, including jogs and junctions (Danna and Benoit, 1993; Rhee et al., 1998; Baskes et al., 1998; Huang et al., 1999; Tang et al., 1999; Rodney and Phillips, 1999; Phillips et al., 1999; Zbib et al., 2000; Shenoy et al., 2000). Experimental estimates of junction strengths have been given by Franciosi and Zaoui (1982) for the 12 slip systems belonging to the family of $\left\{\begin{array}{lll}1 & 1 & 1\end{array}\right\}$ planes and [ 1110$]$ directions in fcc crystals, and by Franciosi (1983) for the

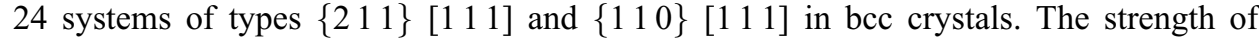
some of these interactions has recently been computed using atomistic and continuum models (Baskes et al., 1998; Rodney and Phillips, 1999; Phillips et al., 1999; Shenoy et al., 2000). Tang et al. (1999) have numerically estimated the average strength of dislocation junctions for $\mathrm{Nb}$ and Ta crystals.

For purposes of the present theory, we specifically concern ourselves with short-range interactions between dislocations which can be idealized as point defects. For simplicity, we consider the case in which each intersecting dislocation acquires a jog. The energy of a pair of crossing dislocations is schematically shown in Fig. 4 as a function of some convenient reaction coordinate, such as the distance between the dislocations. The interaction may be repulsive, resulting in an energy barrier, or attractive, resulting in a binding energy (Fig. 4). In the spirit of an equilibrium theory, here we consider only the final reaction product, corresponding to a pair of jogged dislocations at infinite distance from each other, and neglect the intermediate states along the reaction path. In addition, we deduce the strength of the obstacles directly from the energy supply required to attain the final state, i.e. the jog-formation energy. Despite the sweeping nature of these assumptions, the predicted saturation strengths in multiple slip are in good agreement with experiment (cf. Section 7), which lends some empirical support to the theory.

We estimate the jog formation energy as follows. Based on energy and mobility considerations already discussed, we may expect the preponderance of forest dislocations to be of screw character, and the mobile dislocation segments to be predominantly of edge character. We therefore restrict our analysis to intersections between screw and edge segments. The geometry of the crossing process is schematically shown in Fig. 5. Each dislocation acquires a jog equal to the Burgers vector of the remaining 


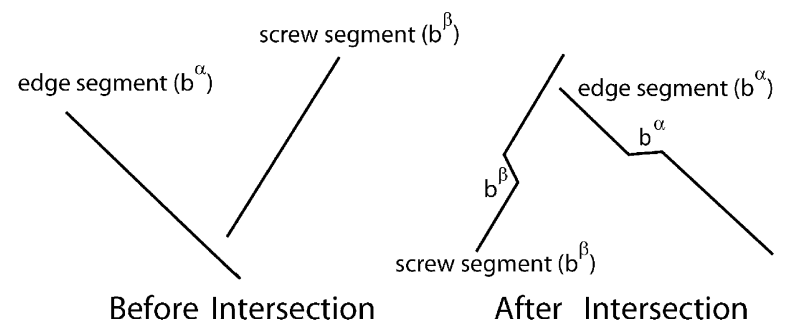

Fig. 5. Schematic of jog formation during dislocation intersection.

dislocation. The energy expended in the formation of the jogs may be estimated as

$$
E_{\alpha \beta}^{\mathrm{jog}} \sim\left|\boldsymbol{b}^{\beta}\right|\left[U^{\alpha \beta}-U^{\alpha \alpha} \cos \theta^{\alpha \beta}\right]+\left|\boldsymbol{b}^{\alpha}\right|\left[U^{\beta \alpha}-U^{\beta \beta} \cos \theta^{\beta \alpha}\right] .
$$

Here, $\alpha$ designates the slip system of the moving edge segment, $\beta$ the slip system of the forest screw dislocation, $U^{\alpha \alpha}=U^{\text {edge }}$ is the energy per unit length of an edge segment in the slip system $\alpha, U^{\beta \beta}=U^{\text {screw }}$ is the energy per unit length of a screw segment in slip system $\beta, U^{\alpha \beta}$ is the energy per unit length of a segment in slip system $\alpha$ aligned with $\boldsymbol{b}^{\beta}, U^{\beta \alpha}$ is the energy per unit length of a segment in slip system $\beta$ aligned with $\boldsymbol{b}^{\alpha}, \theta^{\alpha \beta}$ is the angle between the edge direction in system $\alpha$ and $\boldsymbol{b}^{\beta}$, and $\theta^{\beta \alpha}$ is the angle between the screw direction in system $\beta$ and $\boldsymbol{b}^{\alpha}$.

We additionally assume that the screw orientation defines a cusp in the dependence of the dislocation line energy on segment orientation, and that small deviations of a segment from a pure screw character raise its energy to a level comparable to $U^{\text {edge }}$. This gives the energy estimate

$$
U^{\alpha \beta}=U^{\beta \alpha}= \begin{cases}U^{\text {screw }} & \text { if } \boldsymbol{b}^{\alpha}=\boldsymbol{b}^{\beta}, \\ U^{\text {edge }} & \text { otherwise. }\end{cases}
$$

Inserting these energies into Eq. (46) gives

$$
E_{\alpha \beta}^{\text {jogs }} \sim \begin{cases}b U^{\text {screw }}\left[1-r \cos \theta^{\alpha \beta}\right] & \text { if } \boldsymbol{b}^{\alpha}=\boldsymbol{b}^{\beta}, \\ b U^{\text {screw }}\left[2 r-\cos \left(\theta^{\beta \alpha}\right)-r \cos \theta^{\alpha \beta}\right] & \text { otherwise, }\end{cases}
$$

where $r=U^{\text {edge }} / U^{\text {screw }}$ is the ratio of screw to edge dislocation line energies. For Ta, recent atomistic calculations (Wang et al., 2000) give a value of $r=1.77$. The resulting jog formation energies for the complete collection of pairs of $\{211\}$ and $\left\{\begin{array}{lll}1 & 1 & 0\end{array}\right\}$ dislocations are tabulated in Table 2 .

The net effect of jog formation on hardening may be ascertained as follows. Consider the case in which a primary system $\alpha$ contains forest obstacles of a single species, corresponding to secondary dislocations in the slip system $\beta$. Let $n^{\alpha \beta}$ be the density of forest obstacles per unit area of the primary plane $\alpha$. As a primary dislocation sweeps through an area $A$, the energy expended in forming jogs with forest dislocations of the $\beta$-type is $E_{\alpha \beta}^{\mathrm{jog}} n^{\alpha \beta} A$. On the other hand, the potential energy released as a result of the motion of the dislocation follows from the Peach-Koehler formula as $b \tau^{\alpha} A$, where $\tau^{\alpha}$ is the resolved shear stress acting on the primary system $\alpha$. Hence, the forest 
Table 2

Normalized jog-formation energies resulting from crossings of bcc dislocations

\begin{tabular}{|c|c|c|c|c|c|c|c|c|c|c|c|c|c|c|c|c|c|c|c|c|c|c|c|c|}
\hline & $\mathbf{A 2}$ & $\mathbf{A} \mathbf{2}^{\prime}$ & A3 & $\mathbf{A} 3^{\prime}$ & A6 & $\mathbf{A} \mathbf{6}^{\prime}$ & B2 & $\mathbf{B 2}^{\prime \prime}$ & B4 & $\mathbf{B} 4^{\prime}$ & B5 & $\mathbf{B 5}^{\prime}$ & $\mathrm{C1}$ & $\mathrm{C1}^{\prime}$ & C3 & $\mathbf{C} 3^{\prime \prime}$ & C5 & $\mathbf{C 5 ^ { \prime \prime }}$ & D1 & D1" & D4 & $\mathrm{D4}^{\prime \prime}$ & D6 & D6 $^{\prime \prime}$ \\
\hline A2 & - & 1.0 & 1.0 & 1.0 & 1.0 & 1.0 & 1.5 & 1.5 & 1.5 & 1.5 & 1.5 & 1.5 & 2.4 & 2.4 & 2.4 & 2.4 & 2.4 & 2.4 & 2.4 & 2.4 & 2.4 & 2.4 & 2.4 & 2.4 \\
\hline $\mathbf{A} \mathbf{2}^{\prime}$ & 1.0 & - & 1.0 & 1.0 & 1.0 & 1.0 & 3.2 & 3.2 & 3.2 & 3.2 & 3.2 & 3.2 & 1.8 & 1.8 & 1.8 & 1.8 & 1.8 & 1.8 & 1.8 & 1.8 & 1.8 & 1.8 & 1.8 & 1.8 \\
\hline A3 & 1.0 & 1.0 & - & 1.0 & 1.0 & 1.0 & 2.4 & 2.4 & 2.4 & 2.4 & 2.4 & 2.4 & 1.5 & 1.5 & 1.5 & 1.5 & 1.5 & 1.5 & 2.4 & 2.4 & 2.4 & 2.4 & 2.4 & 2.4 \\
\hline $\mathbf{A 3}^{\prime}$ & 1.0 & 1.0 & 1.0 & - & 1.0 & 1.0 & 1.8 & 1.8 & 1.8 & 1.8 & 1.8 & 1.8 & 3.2 & 3.2 & 3.2 & 3.2 & 3.2 & 3.2 & 1.8 & 1.8 & 1.8 & 1.8 & 1.8 & 1.8 \\
\hline A6 & 1.0 & 1.0 & 1.0 & 1.0 & - & 1.0 & 2.4 & 2.4 & 2.4 & 2.4 & 2.4 & 2.4 & 2.4 & 2.4 & 2.4 & 2.4 & 2.4 & 2.4 & 1.5 & 1.5 & 1.5 & 1.5 & 1.5 & 1.5 \\
\hline $\mathbf{A 6 ^ { \prime }}$ & 1.0 & 1.0 & 1.0 & 1.0 & 1.0 & - & 1.8 & 1.8 & 1.8 & 1.8 & 1.8 & 1.8 & 1.8 & 1.8 & 1.8 & 1.8 & 1.8 & 1.8 & 3.2 & 3.2 & 3.2 & 3.2 & 3.2 & 3.2 \\
\hline B2 & 1.5 & 1.5 & 1.5 & 1.5 & 1.5 & 1.5 & - & 1.0 & 1.0 & 1.0 & 1.0 & 1.0 & 2.4 & 2.4 & 2.4 & 2.4 & 2.4 & 2.4 & 2.4 & 2.4 & 2.4 & 2.4 & 2.4 & 2.4 \\
\hline B2 ${ }^{\prime \prime}$ & 3.2 & 3.2 & 3.2 & 3.2 & 3.2 & 3.2 & 1.0 & - & 1.0 & 1.0 & 1.0 & 1.0 & 1.8 & 1.8 & 1.8 & 1.8 & 1.8 & 1.8 & 1.8 & 1.8 & 1.8 & 1.8 & 1.8 & 1.8 \\
\hline B4 & 2.4 & 2.4 & 2.4 & 2.4 & 2.4 & 2.4 & 1.0 & 1.0 & - & 1.0 & 1.0 & 1.0 & 2.4 & 2.4 & 2.4 & 2.4 & 2.4 & 2.4 & 1.5 & 1.5 & 1.5 & 1.5 & 1.5 & 1.5 \\
\hline $\mathbf{B 4}^{\prime}$ & 1.8 & 1.8 & 1.8 & 1.8 & 1.8 & 1.8 & 1.0 & 1.0 & 1.0 & - & 1.0 & 1.0 & 1.8 & 1.8 & 1.8 & 1.8 & 1.8 & 1 & 3.2 & 3.2 & 3.2 & 3.2 & 3.2 & 3.2 \\
\hline B5 & 2.4 & 2.4 & 2.4 & 2.4 & 2.4 & 2.4 & 1.0 & 1.0 & 1.0 & 1.0 & - & 1.0 & 1.5 & 1.5 & 1.5 & 1.5 & 1.5 & 1.5 & 2.4 & 2.4 & 2.4 & 2.4 & 2.4 & 2.4 \\
\hline $\mathbf{B 5}^{\prime}$ & 1.8 & 1.8 & 1.8 & 1.8 & 1.8 & 1.8 & 1.0 & 1.0 & 1.0 & 1.0 & 1.0 & - & 3.2 & 3.2 & 3.2 & 3.2 & 3.2 & 3.2 & 1.8 & 1.8 & 1.8 & 1.8 & 1.8 & 1.8 \\
\hline C1 & 1.8 & 1.8 & 1.8 & 1.8 & 1.8 & 1.8 & 1.8 & 1.8 & 1.8 & 1.8 & 1.8 & 1.8 & - & 1.0 & 1.0 & 1.0 & 1.0 & 1.0 & 3.2 & 3.2 & 3.2 & 3.2 & 3.2 & 3.2 \\
\hline $\mathrm{C1}^{\prime}$ & 1.8 & 1.8 & 1.8 & 1.8 & 1.8 & 1.8 & 1.8 & 1.8 & 1.8 & 1.8 & 1.8 & 1.8 & 1.0 & - & 1.0 & 1.0 & 1.0 & 1.0 & 3.2 & 3.2 & 3.2 & 3.2 & 3.2 & 3.2 \\
\hline $\mathrm{C} 3$ & 1.5 & 1.5 & 1.5 & 1.5 & 1.5 & 1.5 & 2.4 & 2.4 & 2.4 & 2.4 & 2.4 & 2.4 & 1.0 & 1.0 & - & 1.0 & 1.0 & 1.0 & 2.4 & 2.4 & 2.4 & 2.4 & 2.4 & 2.4 \\
\hline $\mathrm{C}^{\prime \prime}$ & 3.2 & 3.2 & 3.2 & 3.2 & 3.2 & 3.2 & 1.8 & 1.8 & 1.8 & 1.8 & 1.8 & 1.8 & 1.0 & 1.0 & 1.0 & - & 1.0 & 1.0 & 1.8 & 1.8 & 1.8 & 1.8 & 1.8 & 1.8 \\
\hline C5 & 2.4 & 2.4 & 2.4 & 2.4 & 2.4 & 2.4 & 1.5 & 1.5 & 1.5 & 1.5 & 1.5 & 1.5 & 1.0 & 1.0 & 1.0 & 1.0 & - & 1.0 & 2.4 & 2.4 & 2.4 & 2.4 & 2.4 & 2.4 \\
\hline $\mathrm{C5}^{\prime \prime}$ & 1.8 & 1.8 & 1.8 & 1.8 & 1.8 & 1.8 & 3.2 & 3.2 & 3.2 & 3.2 & 3.2 & 3.2 & 1.0 & 1.0 & 1.0 & 1.0 & 1.0 & - & 1.8 & 1.8 & 1.8 & 1.8 & 1.8 & 1.8 \\
\hline D1 & 1.8 & 1.8 & 1.8 & 1.8 & 1.8 & 1.8 & 1.8 & 1.8 & 1.8 & 1.8 & 1.8 & 1.8 & 3.2 & 3.2 & 3.2 & 3.2 & 3.2 & 3.2 & - & 1.0 & 1.0 & 1.0 & 1.0 & 1.0 \\
\hline D1 ${ }^{\prime \prime}$ & 1.8 & 1.8 & 1.8 & 1.8 & 1.8 & 1.8 & 1.8 & 1.8 & 1.8 & 1.8 & 1.8 & 1.8 & 3.2 & 3.2 & 3.2 & 3.2 & 3.2 & 3.2 & 1.0 & - & 1.0 & 1.0 & 1.0 & 1.0 \\
\hline D4 & 2.4 & 2.4 & 2.4 & 2.4 & 2.4 & 2.4 & 1.5 & 1.5 & 1.5 & 1.5 & 1.5 & 1.5 & 2.4 & 2.4 & 2.4 & 2.4 & 2.4 & 2.4 & 1.0 & 1.0 & - & 1.0 & 1.0 & 1.0 \\
\hline $\mathbf{D 4}^{\prime \prime}$ & 1.8 & 1.8 & 1.8 & 1.8 & 1.8 & 1.8 & 3.2 & 3.2 & 3.2 & 3.2 & 3.2 & 3.2 & 1.8 & 1.8 & 1.8 & 1.8 & 1.8 & 1.8 & 1.0 & 1.0 & 1.0 & - & 1.0 & 1.0 \\
\hline D6 & 1.5 & 1.5 & 1.5 & 1.5 & 1.5 & 1.5 & 2.4 & 2.4 & 2.4 & 2.4 & 2.4 & 2.4 & 2.4 & 2.4 & 2.4 & 2.4 & 2.4 & 2.4 & 1.0 & 1.0 & 1.0 & 1.0 & - & 1.0 \\
\hline D6 $^{\prime \prime}$ & 3.2 & 3.2 & 3.2 & 3.2 & 3.2 & 3.2 & 1.8 & 1.8 & 1.8 & 1.8 & 1.8 & 1.8 & 1.8 & 1.8 & 1.8 & 1.8 & 1.8 & 1.8 & 1.0 & 1.0 & 1.0 & 1.0 & 1.0 & - \\
\hline
\end{tabular}

obstacles of type $\beta$ become 'transparent' to the motion of primary dislocations when $b \tau^{\alpha} A \geqslant E_{\alpha \beta}^{\mathrm{jog}} n^{\alpha \beta} A$, or

$$
\tau^{\alpha} \geqslant \frac{1}{b} E_{\alpha \beta}^{\mathrm{jog}} n^{\alpha \beta}
$$

Since the jog energies scale with the elastic moduli, they may be expected to reduce to zero at the melting temperature, which to some extent accounts for the observed thermal softening. In addition, the jog energies are small enough for thermal activation to be operative at the level of individual obstacles. A derivation entirely analogous to that leading to Eq. (14) yields, in this case,

$$
\frac{s^{\alpha \beta}}{s_{0}^{\alpha \beta}}=\frac{1}{\beta E_{\alpha \beta}^{\mathrm{jog}}} \mathrm{a} \sinh \left(\frac{\dot{\gamma}^{\alpha}}{\gamma_{0}^{\alpha}} \mathrm{e}^{\beta_{\alpha \beta}^{\mathrm{jog}}}\right),
$$

where

$$
s_{0}^{\alpha \beta}=\frac{E_{\alpha \beta}^{\mathrm{jog}}}{b \bar{l}^{\alpha} L^{\mathrm{junct}}}
$$

and

$$
\dot{\gamma}_{0}^{\alpha}=2 \rho^{\alpha} b \bar{l}^{\alpha} v_{\mathrm{D}}
$$




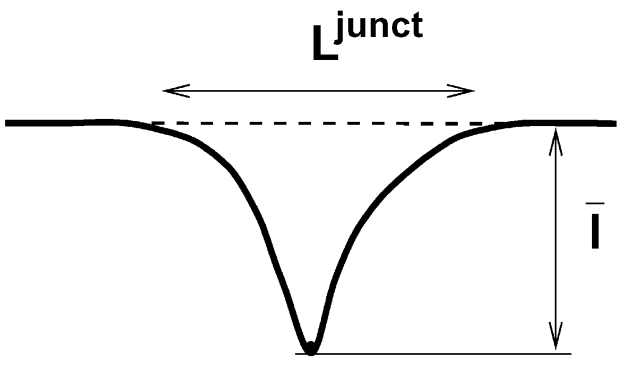

Fig. 6. Schematic of a dislocation line overcoming a junction.

The lengths $\bar{l}^{\alpha}$ and $L^{\text {junct }}$ describe the geometry of the junction, as illustrated in Fig. 6, and are of the order of a few Burgers vectors.

It follows from these developments that, at low temperatures, the energies collected in Table 2 provide a measure of the corresponding obstacle strengths. We may also recall that the saturation flow stress of a slip system is directly related to the strengths of the obstacles. Consequently, information about obstacle strengths may be inferred from saturation flow stresses in crystals deformed in double slip. Measurements of this type have been performed by Franciosi for $\alpha$-Fe (Franciosi, 1983). The general trends exhibited by Table 2 are consistent with Franciosi's data, as well as with the recent analytical model of Lee et al. (1999). In addition, the saturation flow stresses resulting from the model developed above are in good quantitative agreement with those measured experimentally (cf. Section 7) which lends empirical support to jog-formation as a plausible mechanism underlying short-range obstacle strength and saturation in bcc crystals.

\section{Dislocation evolution}

The density of forest obstacles depends directly on the dislocation densities in all slip systems of the crystal. Therefore, in order to close the model we require an equation of evolution for the dislocation densities. Processes resulting in changes in dislocation density include production by fixed sources, such as Frank-Read sources, breeding by double cross slip and pair annihilation (see Kuhlmann-Wilsdorf (1989) for a review; see also Johnston and Gilman (1959, 1960); Gillis and Gilman (1965); Essmann and Rapp (1973); Lagerlof (1993); Dybiec (1995)). Although the operation of fixed Frank-Read sources is quickly eclipsed by production due to cross-slip at finite temperatures, it is an important mechanism at low temperatures. The double cross-slip, fixed Frank-Read sources and pair annihilation mechanisms are next considered in turn.

\subsection{Breeding by double cross-slip}

The importance of breeding by double cross-slip as a dislocation generation mechanism in crystals was emphasized by Johnston and Gilman $(1959,1960)$. In this mecha- 
nism, the screw section of a moving dislocation migrates to a parallel plane by double cross-slip, thus creating a pair of sessile segments which pin down the dislocation and act in a manner similar to a Frank-Read source.

The rate at which dislocation length is produced by this mechanism can be estimated as follows. Let $N_{\text {CS }}^{\alpha}$ denote the number of dynamic sources per unit volume induced by double cross-slip on slip system $\alpha$. Additionally, let $L^{\text {sat }}$ denote the dislocation length emitted by each source prior to saturation. The rate of dislocation production per unit volume is, therefore

$$
\dot{\rho}_{\mathrm{CS}}^{\alpha}=L^{\mathrm{sat}} \dot{N}_{\mathrm{CS}}^{\alpha}
$$

Assuming that cross-slip is thermally activated (see, e.g., Rasmussen et al. (1997); Rao et al. (1999) for recent calculations of pathways and energy barriers in copper), the rate $\dot{N}_{\text {CS }}^{\alpha}$ at which dynamic sources are generated may in turn be computed as follows. Let $\bar{L}$ be the mean-free path between cross-slip events, $L^{\text {cross }}$ the length of the screw segment effecting the double cross glide, and $E^{\text {cross }}$ the energy barrier for cross-slip. The frequency of cross glide attempts per unit volume is, therefore, $\rho^{\alpha} v^{\alpha} /\left(\bar{L} L^{\text {cross }}\right)$, where $v^{\alpha}$ is the mean dislocation velocity. According to transition-state theory, of these attempts a fraction $\mathrm{e}^{-\beta E^{\text {cross }}}$ is successful. This gives

$$
\dot{N}_{\mathrm{CS}}^{\alpha}=\frac{\rho^{\alpha} v^{\alpha}}{\bar{L} L^{\text {cross }}} \mathrm{e}^{-\beta E^{\text {cross }}} \text {. }
$$

Indeed, theoretical ( $\mathrm{Li}, 1961)$ and experimental (Johnston and Gilman, 1959, 1960) investigations suggest that the breeding rate due to cross-glide is proportional to the mean dislocation speed. Using Orowan's formula, Eq. (54) may be rewritten as

$$
\dot{N}_{\mathrm{CS}}^{\alpha}=\frac{\dot{\gamma}^{\alpha}}{b \bar{L} L^{\text {cross }}} \mathrm{e}^{-\beta E^{\text {cross }}} \text {. }
$$

Inserting this relation into Eq. (53) gives

$$
\dot{\rho}_{\mathrm{CS}}^{\alpha}=\frac{L^{\mathrm{sat}} \dot{\gamma}^{\alpha}}{b \bar{L} L^{\text {cross }}} \mathrm{e}^{-\beta E^{\text {cross }}}
$$

Following Essmann and Rapp (1973), we shall additionally assume that the mean free path is inversely proportional to the dislocation density, which gives

$$
b \dot{\rho}_{\mathrm{CS}}^{\alpha}=\lambda_{\mathrm{CS}} \sqrt{\rho^{\alpha}} \dot{\gamma}^{\alpha}
$$

where we have written

$$
\lambda_{\mathrm{CS}}=\frac{L^{\mathrm{sat}}}{L^{\text {cross }}} \mathrm{e}^{-\beta E^{\text {cross }}} .
$$

An improvement on this model is to account for the resolved shear stress acting on the cross-slip plane in the computation of the activation energy, resulting in a socalled non-Schmid effect, but this possibility will not be pursued here in the interest of simplicity.

The double cross-slip mechanism is geometrically similar to the double kink formation alluded to earlier. By virtue of this similarity, we may expect that $L^{\text {cross }} \approx L^{\text {kink }}$ and $E^{\text {cross }} \approx E^{\text {kink }}$ to a first approximation. 


\subsection{Multiplication by fixed Frank-Read sources}

An infinite amount of dislocation multiplication can be sustained by the fixed FrankRead sources as long as newly formed loops can expand and move away from the pinning points. The precise details of this mechanism, which was independently proposed by Frank and Read (1950) in the early 1950s, are well documented and can be consulted elsewhere (Hirth and Lothe, 1968). Here, we limit our analysis to obtain an estimate for the dislocation multiplication based on this mechanism. Considering that the obstacles in the slip plane $\alpha$ serve as pinning points to operate the Frank-Read sources, the increase of the dislocation population is given by

$$
\dot{\rho}_{\mathrm{FR}}^{\alpha}=L_{\mathrm{FR}} \dot{N}_{\mathrm{FR}}^{\alpha},
$$

where $N_{\mathrm{FR}}^{\alpha}$ is the number of active sources per unit volume and $L_{\mathrm{FR}}^{\alpha}$ is the length of the emitted segment by the source and $n^{\alpha}$ is the obstacle density given by Eq. (25). The effective rate of activation of Frank-Read sources in a given system $\alpha$ can be estimated by the number of intersections of the gliding loops with obstacles in that system weighted by the efficiency of the source. In order to account for the possibility of the newly formed loops to glide away from the obstacles, we introduce the factor $\sqrt{\rho^{\alpha} / n^{\alpha}}$ which is the ratio between mean distance between the obstacles and the mean dislocation spacing. Then,

$$
\dot{N}_{\mathrm{FR}}^{\alpha}=\sqrt{\frac{\rho^{\alpha}}{n^{\alpha}}} n^{\alpha} v^{\alpha} \rho^{\alpha} .
$$

Introducing Orowan's formula and assuming that the length of dislocation segments is proportional to the mean distance between obstacles, the multiplication rate due to fixed Frank-Read sources can be written as

$$
b \dot{\rho}_{\mathrm{FR}}^{\alpha}=\lambda_{\mathrm{FR}} \sqrt{\rho^{\alpha}} \dot{\gamma}^{\alpha} .
$$

Unlike breeding by cross-glide, dislocation multiplication by fixed Frank-Read sources is not a thermally activated process, and thus, it remains operative even at low temperatures. At finite temperatures, however, both mechanisms contribute to the total dislocation multiplication rate.

It should be pointed out that both Eqs. (57) and (61) predict that $\rho^{\alpha}$ grows as $\left(\gamma^{\alpha}\right)^{2}$. This rate of growth is indeed observed in many crystals during the intermediate stages of dislocation multiplication.

\subsection{Attrition by pair annihilation}

Dislocation densities are often observed to attain a saturation density at sufficiently large strains (Ashby, 1972). This saturation stage arises as a result of the competition between dislocation multiplication mechanisms such as double cross-slip and pair annihilation. For instance, spontaneous annihilation is observed in metals such as copper for screw dipole heights less than $1 \mathrm{~nm}$ (Essmann and Mughrabi, 1979). Pair annihilation is mainly the result of the cross-slip of screw segments of opposite sign (Arias and Joannopoulos, 1994; Wang et al., 2000; Rasmussen et al., 2000). 


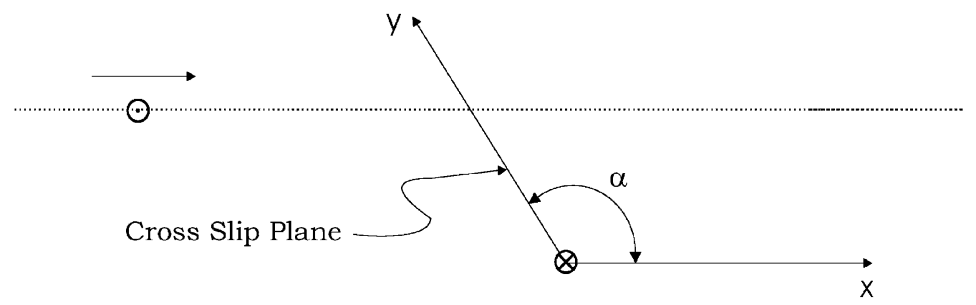

Fig. 7. Interaction between two parallel screw dislocations of opposite sign moving on parallel slip planes.

Huang et al. (1999), have studied the dynamic stability of short-range linear-elastic interactions between two dislocations of parallel line vectors which glide on two parallel slip planes in bcc crystals. Here, we develop a similar but somewhat simpler linear-elastic model of dislocation pair annihilation. Thus, our goal is to estimate the frequency with which two parallel screw segments moving on parallel planes will acquire converging trajectories leading to their mutual annihilation. We note that, for simplicity, our analysis is restricted to annihilation between pairs of dislocations belonging to the same slip system.

Consider a screw segment moving under the action of an applied shear stress $\tau$. The segment follows a path which brings it in close proximity to a second immobile screw segment, (Fig. 7). Let $(x, y)$ be coordinates centered at the fixed dislocation such that $x$ points in the direction of motion of the incoming dislocation and $y$ points in the direction of the cross-slip plane (Fig. 7). The interaction force per unit length exerted on the moving segment along the cross-slip plane is (Hirth and Lothe, 1968)

$$
f_{y}=-K \frac{y}{r^{2}}
$$

where $K$ is the pre-logarithmic factor for a screw segment, and

$$
r=\sqrt{x^{2}+y^{2}+2 x y \cos \alpha}
$$

where $\alpha$ is the angle subtended by the $x$ and $y$ directions (Fig. 7). For bec crystals, $\alpha=2 \pi / 3$. For isotropic crystals, $K=\mu b^{2} / 4 \pi$. In view of Eq. (11), the equation of motion for the incoming screw dislocation is

$$
\dot{y}=2 l_{\mathrm{P}} v_{\mathrm{D}} \mathrm{e}^{-\beta E^{\text {cross }}} \sinh \left[\beta E^{\text {cross }} \frac{\left(K y / b r^{2}\right)}{\tau_{0}}\right]
$$

for $y>\kappa_{0}>0$, where the characteristic distance

$$
\kappa_{0}=\frac{K}{b \tau_{0}}
$$

is determined by the condition $f_{y}=b \tau_{0}$. For $y<\kappa_{0}$ dislocation motion is no longer a thermally activated process and therefore Eq. (64), which is based on the transition-state theory, does no longer apply. In this regime, where the interaction force exceeds the Peierls barrier, dislocation mobility increases significantly (Suzuki et al., 1991).

Depending on the value of $y$ as $x \rightarrow-\infty$, the moving segment bypasses or is captured by the second segment. The precise calculation of the trajectories of the moving dislocation requires numerical computation. However, the essence of the annihilation 
mechanism can be captured by the following simple argument. Consider a dislocation initially at rest at position $\left(x=0, y_{0}\right)$. Subsequently, the motion of the dislocation for $y \geqslant \kappa_{0}$ is governed by the equation

$$
\dot{y}=-2 l_{\mathrm{P}} v_{\mathrm{D}} \mathrm{e}^{-\beta E^{\text {cross }}} \sinh \left(\beta E^{\text {cross }} \frac{(K / b y)}{\tau_{0}}\right) .
$$

This equation is separable, and the time required for the annihilation process follows simply as

$$
t=\int_{\kappa_{0}}^{y_{0}}\left\{2 l_{\mathrm{P}} v_{\mathrm{D}} \mathrm{e}^{-\beta E^{\text {cross }}} \sinh \left(\beta E^{\text {cross }} \frac{(K / b y)}{\tau_{0}}\right)\right\}^{-1} \mathrm{~d} y .
$$

In order to facilitate this calculation, the time required to travel from $y=\kappa_{0}$ to 0 is neglected in the previous equation due to the much higher dislocation mobility in this regime and the small value of $\kappa_{0}$, of the order of few $b$ for bcc crystals. Additionally, we may simply set $\sinh (x) \approx x$ to a first approximation, with the result:

$$
t=\frac{\mathrm{e}^{\beta E^{\text {cross }}}}{2 l_{\mathrm{P}} v_{\mathrm{D}} \beta E^{\text {cross }}} \frac{y_{0}^{2}-\kappa_{0}^{2}}{2 \kappa_{0}} .
$$

On the other hand, the time which the incoming dislocation spends at distances of the order of $y_{0}$ to the receiving dislocation is

$$
t \sim \frac{b \rho^{\alpha}}{\dot{\gamma}^{\alpha}} y_{0}
$$

For annihilation to be possible, the annihilation time (68) must be less than the time (69) which the incoming dislocation spends in the immediate vicinity of the target dislocation. This yields the condition

$$
y_{0} \leqslant \kappa=\kappa_{0}\left(A+\sqrt{A^{2}+1}\right),
$$

where

$$
A=\mathrm{e}^{-\beta E^{\text {cross }}} \beta E^{\text {cross }} \dot{\gamma}_{0}^{\text {cross }} / \dot{\gamma}^{\alpha}
$$

is a factor depending on the strain rate and temperature,

$$
\dot{\gamma}_{0}^{\text {cross }}=2 b \rho l_{\mathrm{P}} v_{\mathrm{D}}
$$

is a reference slip-strain rate, and $\kappa$ may now be regarded as an effective pair annihilation distance. The cut-off value $\kappa_{\mathrm{c}}$, corresponding to the maximal pair annihilation distance, is the effective screening distance which can be set equal to the mean distance between dislocations.

A simple expression which interpolates between the extreme values of $\kappa$ is

$$
\frac{1}{\kappa}=\frac{1}{\kappa_{\mathrm{c}}}+\frac{1}{\kappa_{0}\left(A+\sqrt{A^{2}+1}\right)} .
$$

It follows that the critical pair-annihilation distance $\kappa$ decreases with increasing strain rate and decreasing temperature. Thus, at high strain rates the dislocation velocities are high and the probability of being captured by another dislocation diminishes accordingly. Additionally, an increase in temperature increases the dislocation mobility and 
speeds up the annihilation process, which results in an attendant increase in annihilation rates. As will be demonstrated in Section 7, these trends are important in order to capture the temperature and strain-rate dependence of the stage I-II transition in Ta.

The rate of dislocation attrition due to pair annihilation may finally be estimated as follows. Over a differential of time $\mathrm{d} t$, the number of annihilation events per unit volume is

$$
\mathrm{d} N^{\alpha}=\frac{1}{2 L^{\text {cross }}}\left(\rho^{\alpha}\right)^{2} \kappa v^{\alpha} \mathrm{d} t .
$$

The attendant loss of dislocation length per unit volume is

$$
\mathrm{d} \rho^{\alpha}=-2 L^{\text {cross }} \mathrm{d} N^{\alpha} .
$$

Combining these relations and using Orowan's formula, the rate of pair annihilation may finally be expressed in the form

$$
b \dot{\rho}^{\alpha}=-\kappa \rho^{\alpha} \dot{\gamma}^{\alpha} .
$$

\subsection{Dislocation multiplication rate}

Combining Eqs. (57), (61) and (76), the total rate of change of the dislocation density follows as

$$
b \dot{\rho}^{\alpha}=\lambda \sqrt{\rho^{\alpha}} \dot{\gamma}^{\alpha}-\kappa \rho^{\alpha} \dot{\gamma}^{\alpha},
$$

where $\lambda=\lambda_{\mathrm{CS}}+\lambda_{\mathrm{FR}}$. Evidently, $\dot{\rho}^{\alpha}=0$ upon the attainment of the saturation density:

$$
\rho^{\text {sat }}=\left(\frac{\lambda}{\kappa}\right)^{2} .
$$

Dividing through by $\rho^{\text {sat }}$, Eq. (78) may be recast in the form:

$$
\frac{\dot{\rho}^{\alpha}}{\rho^{\text {sat }}}=\left(\sqrt{\frac{\rho^{\alpha}}{\rho^{\text {sat }}}}-\frac{\rho^{\alpha}}{\rho^{\text {sat }}}\right) \frac{\dot{\gamma}^{\alpha}}{\gamma^{\text {sat }}},
$$

where

$$
\gamma^{\text {sat }}=\frac{b}{\kappa}
$$

is a saturation slip strain. Eq. (79) can be integrated, yielding

$$
\frac{\rho^{\alpha}}{\rho^{\mathrm{sat}}}=\left[1+\left(\sqrt{\frac{\rho_{0}^{\alpha}}{\rho^{\mathrm{sat}}}}-1\right) \exp \left(-\frac{1}{2} \frac{\gamma^{\alpha}}{\gamma^{\mathrm{sat}}}\right)\right]^{2} .
$$

The rate equation (79) expresses a competition between the dislocation multiplication and annihilation mechanisms. For slip strains $\gamma^{\alpha} \ll \gamma^{\text {sat }}$, the multiplication term dominates and, as noted previously, the dislocation density $\dot{\rho}^{\alpha}$ grows as $\left(\dot{\gamma}^{\alpha}\right)^{2}$. By contrast, when $\gamma^{\alpha} \gg \gamma^{\text {sat }}$ the rates of multiplication and annihilation balance out and saturation sets in. After saturation is attained, the dislocation density remains essentially unchanged. It should be carefully noted, that, in view of Eq. (73), the saturation slip strain $\gamma_{\text {sat }}$ is a function of temperature and strain rate. In particular, $\gamma_{\text {sat }}$ decreases with increasing 
temperature and decreasing strain rate. Since the stage I-II transition strain scales roughly with $\gamma_{\text {sat }}$, we expect these trends to be exhibited by the transition strain itself, in accordance with experimental observation.

\section{Comparison with experiment}

We proceed to validate the theory against the uniaxial tests on Ta single crystals of Mitchell and Spitzig (1965). In these tests, 99.97\%-pure Ta specimens were loaded in tension along the [213] crystallographic axis, at various combinations of temperature and strain rate. In particular we considered temperatures ranging from 296 to $573 \mathrm{~K}$, and strain rates ranging from $10^{-1}$ to $10^{-5} \mathrm{~s}^{-1}$. The numerical procedure employed for the integration of the constitutive equations has been described elsewhere (Ortiz and Stainier, 1999). The constitutive update is fully implicit, with the active systems determined iteratively so as to minimize an incremental work function. All stress-strain curves are reported in terms of nominal stress and engineering strain.

The material property set used in calculations is collected in Table 3 . The elastic moduli $C_{11}, C_{12}$ and $C_{44}$ were obtained by fitting to the tables of Simmons and Wang (1971). The ratio between edge and screw dislocation-line energies $\left(U^{\text {edge }} / U^{\text {screw }}\right)$ is taken from the atomistic calculations of Wang et al. (2000). The remaining parameters

Table 3

Parameter set for Tantalum

\begin{tabular}{lll}
\hline Parameter & Value & Units \\
\hline$C_{11}$ & $266.49-0.021 T^{\mathrm{a}}$ & $(\mathrm{GPa})$ \\
$C_{12}$ & $156.25-0.006 T$ & $(\mathrm{GPa})$ \\
$C_{44}$ & $90.02-0.015 T$ & $(\mathrm{GPa})$ \\
$b$ & $2.86 \times 10^{-10}$ & $(\mathrm{~m})$ \\
$E^{\text {kink }}$ & 0.70 & $(\mathrm{eV})$ \\
$L^{\text {kink }} / b$ & 13 & \\
$U^{\text {edge }} / \mu b^{2 \mathrm{~b}}$ & 0.216 & \\
$U^{\text {edge }} / U^{\text {screw }}$ & 1.77 & \\
$\bar{l}^{\prime} b$ & 5 & \\
$L^{\text {junct }} / b$ & 20 & $(\mathrm{eV})$ \\
$E^{\text {cross }}$ & 0.67 & \\
$L^{\text {cross }} / b$ & 13 & \\
$\kappa_{c} / b$ & 1250 & $\left(\mathrm{~m}{ }^{-2}\right)$ \\
$\lambda_{\text {FR }}$ & 2.3 & $(\mathrm{MPa})$ \\
$\rho_{0}$ & $10^{12}$ & \\
$g_{0}$ & 8.0 & \\
$a_{0}$ & 0.01 & \\
\hline${ }^{\mathrm{a}} T$ in Kelvin. & \\
${ }^{\mathrm{b}} \mu=\frac{3}{5} C_{44}+\frac{1}{5}\left(C_{11}-C_{12}\right)$. &
\end{tabular}




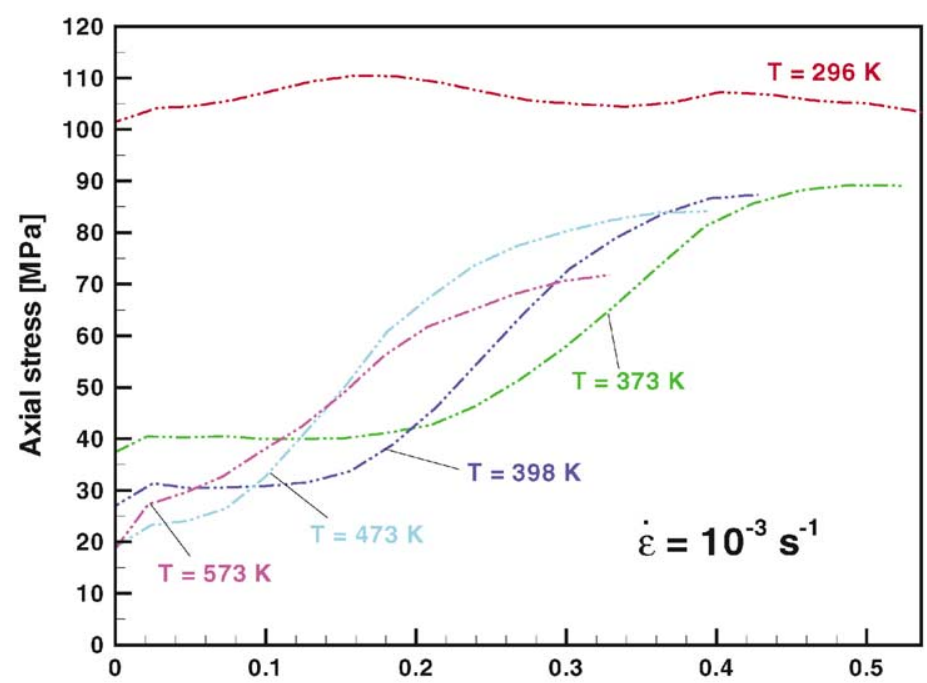

(a)

\section{Axial strain}

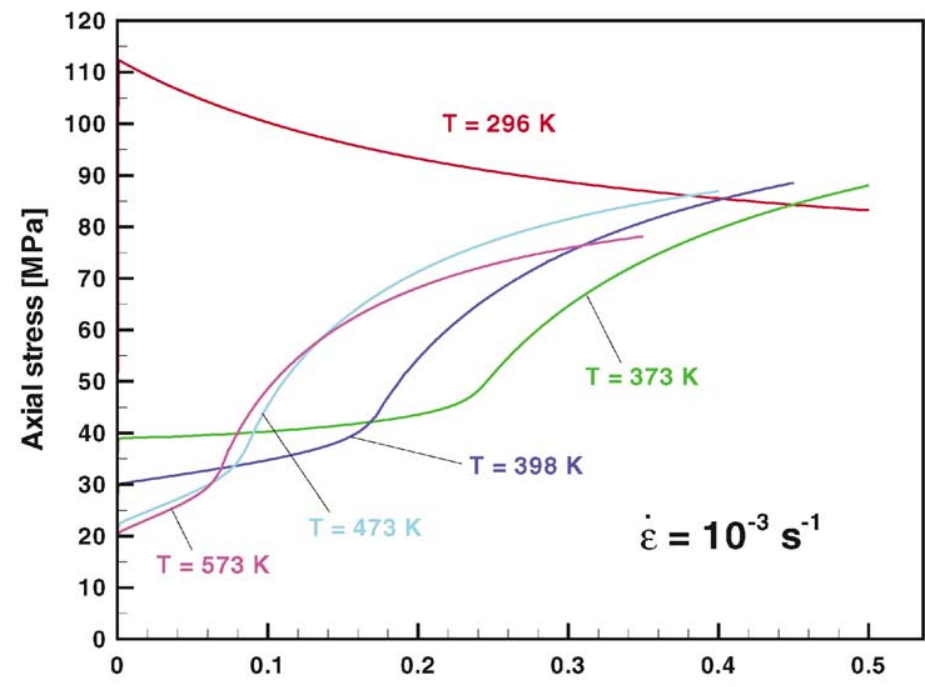

(b)

Axial strain

Fig. 8. Temperature dependence of stress-strain curves for [213] Ta single crystal $\left(\dot{\varepsilon}=10^{-3} \mathrm{~s}^{-1}\right)$. (a) Experimental data of Mitchell \& Spitzig (Mitchell and Spitzig, 1965). (b) Predictions of the model.

have been obtained by fitting to the experimental data of Mitchell and Spitzig (1965). Note that we do not give any value for $L^{\text {sat }}$, since it has no measurable influence in the range of temperatures considered here. It is expected, however, to play a role at higher temperatures.

Figs. 8 and 9 show the predicted and measured stress-strain curves for a [213] Ta crystal over a range of temperatures and strain rates. It is evident from these figures 


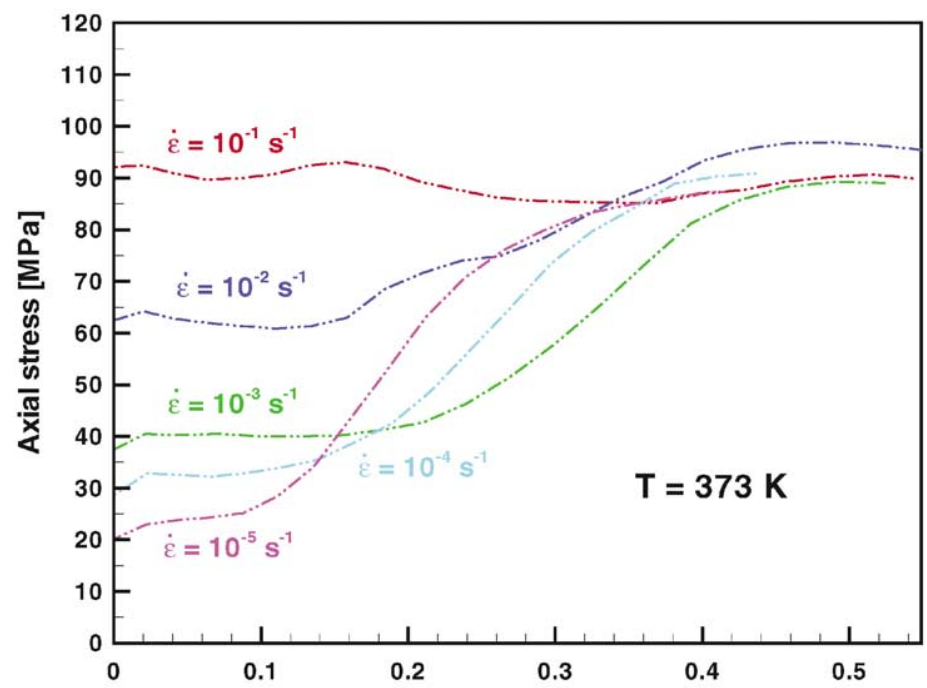

(a)

Axial strain

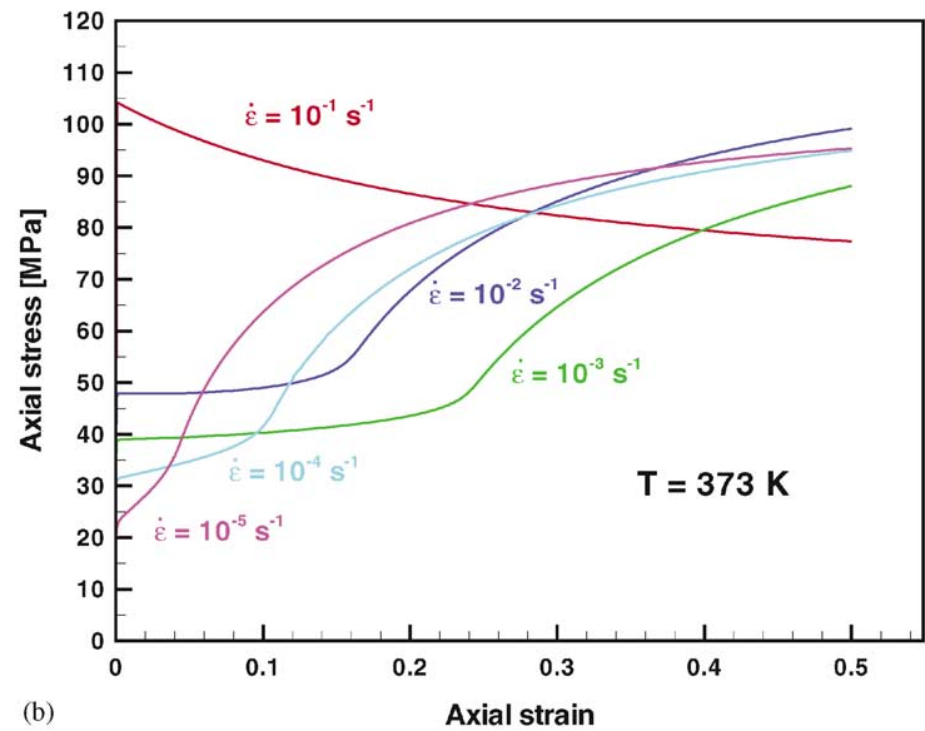

Fig. 9. Strain-rate dependence of stress-strain curves for [2 13] Ta single crystal ( $T=373 \mathrm{~K})$. (a) Experimental data of Mitchell \& Spitzig (Mitchell and Spitzig, 1965). (b) Predictions of the model.

that the model captures salient features of the behavior of Ta crystals such as the dependence of the initial yield point on temperature and strain rate, the presence of a marked stage I of easy glide, specially at low temperature and high strain rates, the sharp onset of stage II hardening and its tendency to shift towards lower strains, and eventually disappear, as the temperature increases or the strain rate decreases, the parabolic stage II hardening at low strain rates or high temperatures, the stage II 


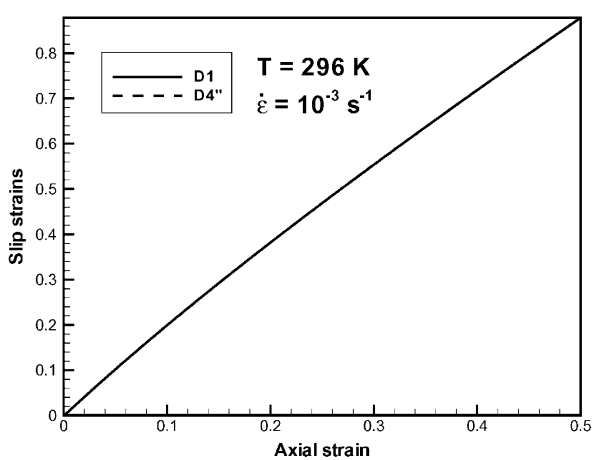

(a) $T=296 \mathrm{~K}$

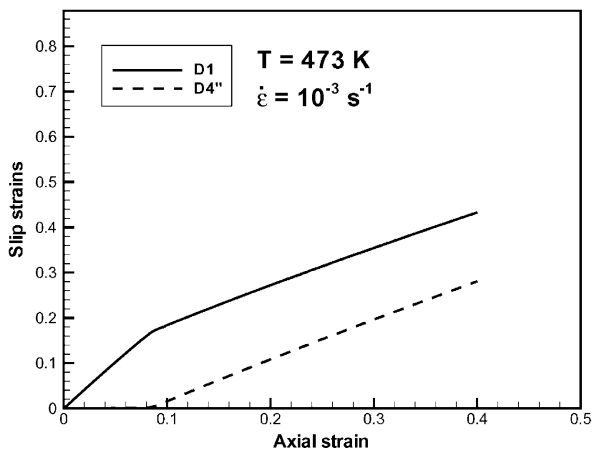

(c) $T=473 \mathrm{~K}$

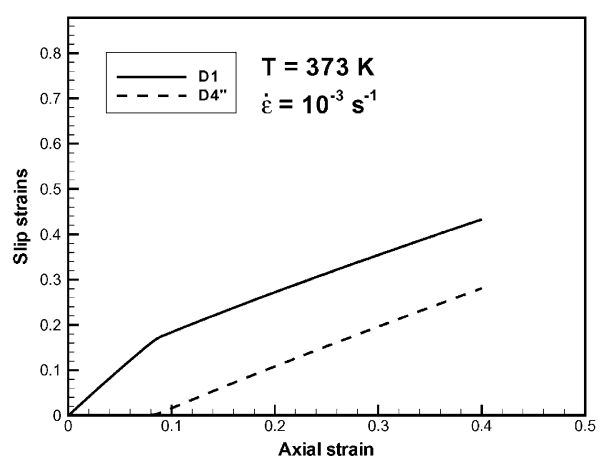

(b) $T=373 \mathrm{~K}$

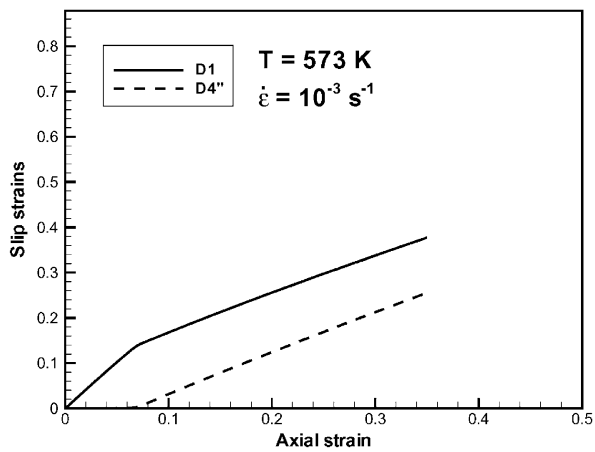

(d) $T=573 K$

Fig. 10. Slip activity as a function of temperature $\left(\dot{\varepsilon}=10^{-3} \mathrm{~s}^{-1}\right)$.

softening at high strain rates or low temperatures; the trend towards saturation at high strains, and the temperature and strain-rate dependence of the saturation stress.

The theory reveals useful insights into the mechanisms underlying these behaviors. For instance, since during stage I the crystal deforms in single slip and the secondary dislocation densities are low, the Peierls resistance dominates and the temperature and strain-rate dependency of yield owes mainly to the thermally activated formation of kinks and crossing of forest dislocations. It is interesting to note that during this stage the effect of increasing (decreasing) temperature is similar to the effect of decreasing (increasing) strain rate, as noted by Tang et al. (1999). The onset of stage II is due to the activation of secondary systems. The rate at which these secondary systems harden during stage I depends on the rate of dislocation multiplication in the primary system. This rate is in turn sensitive to the saturation strain $\gamma^{\text {sat }}$, which increases with strain rate and decreases with temperature. As a result, the length of the stage I of hardening is predicted to increase with strain rate and decrease with temperature, as observed experimentally. Finally, the saturation stress is mainly governed by the forest hardening mechanism and, in particular, by the strength of the forest obstacles. This process is less thermally activated than the Peierls stress, since the corresponding energy barriers 


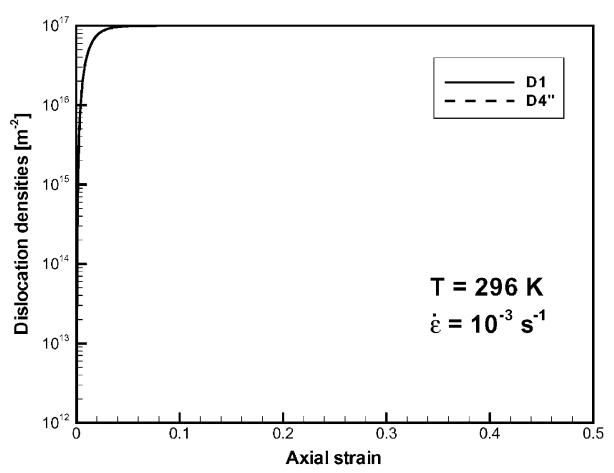

(a) $T=296 \mathrm{~K}$

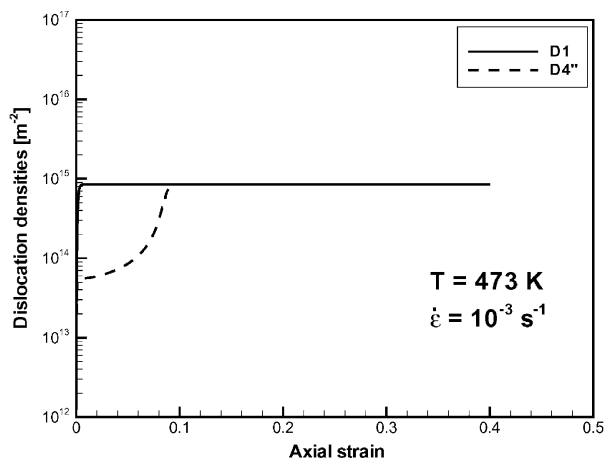

(c) $T=473 \mathrm{~K}$

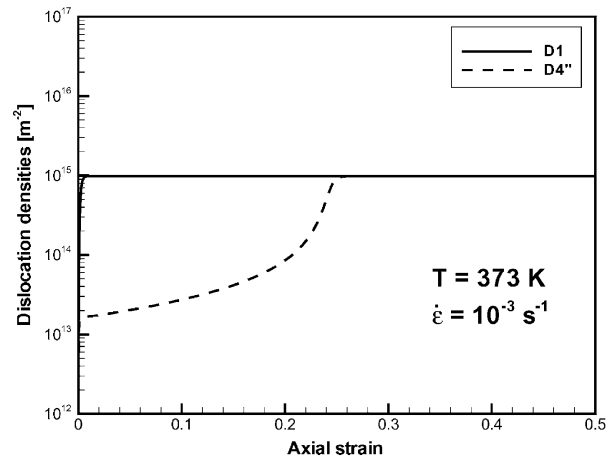

(b) $T=373 \mathrm{~K}$

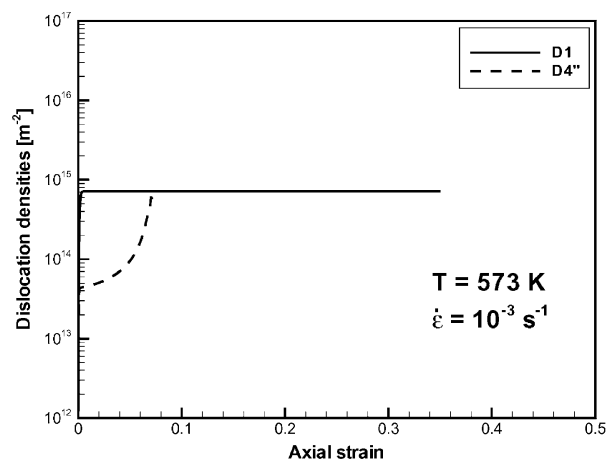

(d) $T=573 \mathrm{~K}$

Fig. 11. Evolution of dislocation densities as a function of temperature $\left(\dot{\varepsilon}=10^{-3} \mathrm{~s}^{-1}\right)$.

are comparatively higher. Consequently, the stress-strain curves tend to converge in this regime, in keeping with observation.

The apparent softening observed in simulation results at the lowest temperature $(296 \mathrm{~K})$ and the highest strain rate $\left(10^{-1} \mathrm{~s}^{-1}\right)$ is actually an effect of the boundary conditions, which allow for some level of rotation of the specimen. Since in those cases, the material hardening is relatively low (stage I only), this geometrical softening dominates the apparent macroscopic behavior. In the other cases, the activation of several systems limits the extend of the rotations reducing the effects of the macroscopic hardening. In order to simulate more precisely the experimental boundary conditions, a model of the entire specimen allowing for a non-homogeneous deformation field should be considered.

The effect of temperature and strain-rate on hardening is also illustrated by the evolution of slip activity in the primary and secondary slip systems, $D 1$ and $D 4^{\prime \prime}$, respectively. Figs. 10 and 11 show the evolution of slip strains and dislocation densities as a function of temperature. It is evident from these figures that dislocation density saturation in the primary system occurs earlier as the temperature is increased, resulting 


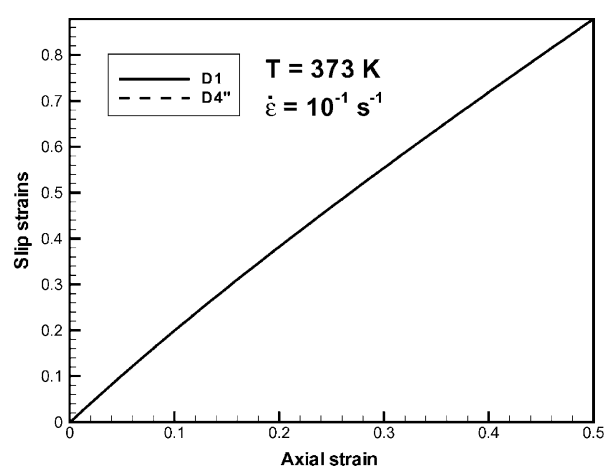

(a) $\dot{\epsilon}=10^{-1} s^{-1}$

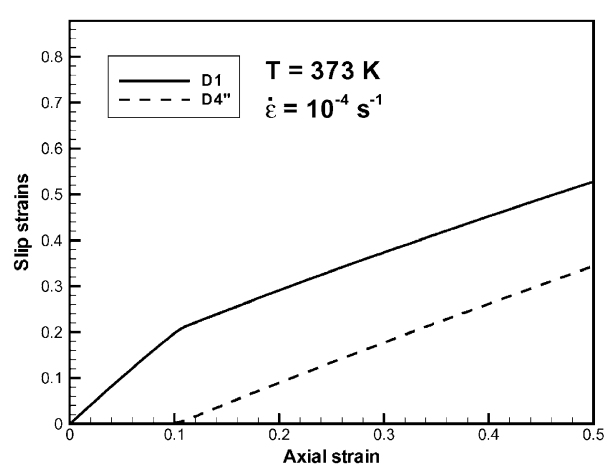

(c) $\dot{\epsilon}=10^{-4} s^{-1}$

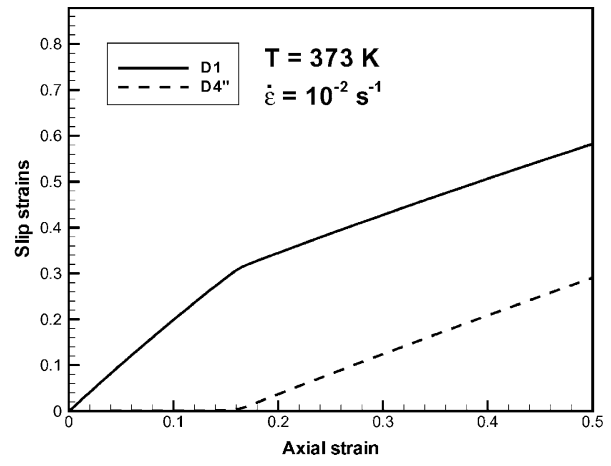

(b) $\dot{\epsilon}=10^{-2} s^{-1}$

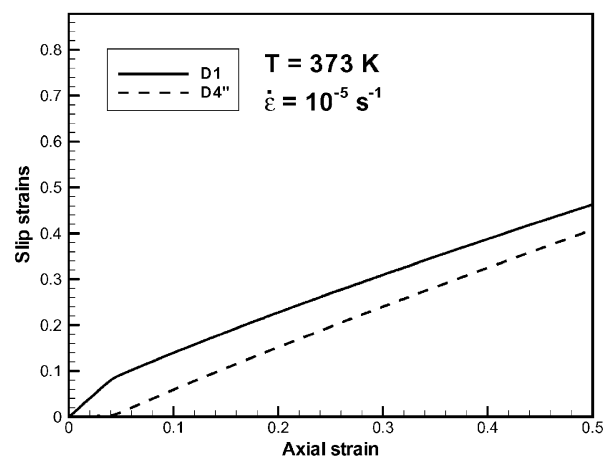

(d) $\dot{\epsilon}=10^{-5} s^{-1}$

Fig. 12. Slip activity as a function of strain-rate $(T=373 \mathrm{~K})$.

in the activation of the secondary system and in the onset of stage II at lower strains. A similar effect is observed when the strain rate is decreased, Figs. 12 and 13.

The effect of loading direction on the hardening rate is illustrated in Fig. 14. The figure shows the stress-strain curves obtained by loading the crystal in the [2 13], [ $\left.\begin{array}{lll}1 & 0 & 1\end{array}\right]$ and [ 1111 l] directions at a strain rate of $10^{-3} \mathrm{~s}^{-1}$ and a temperature of $373 \mathrm{~K}$. Each of these loading directions results in the activation of a different set of slip systems. As may be observed in the figure, the higher initial yield stress in the [111] direction relative to the baseline [213] direction, and the initial negative hardening rate, are fairly well captured by the model. The experimental curve exhibits a subsequent upturn, most likely due to the activation of additional secondary systems, which is not captured by the model. Loading in the [101] direction results in the activation of a large number of systems from the outset and the rate of hardening is correspondingly high. The model appears to over-predict the rate of hardening.

However, as was noted in Cuitiño and Ortiz (1992), the stress-strain curve for crystals loaded in high-symmetry orientations are extremely sensitive to small misalignments in the loading axis, which accounts for the large experimental scatter 


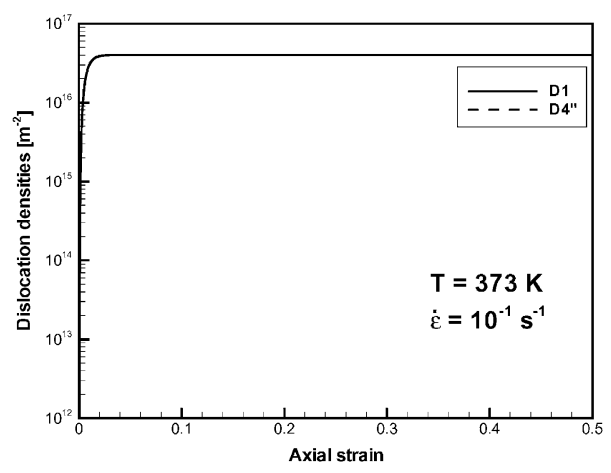

(a) $\dot{\epsilon}=10^{-1} s^{-1}$

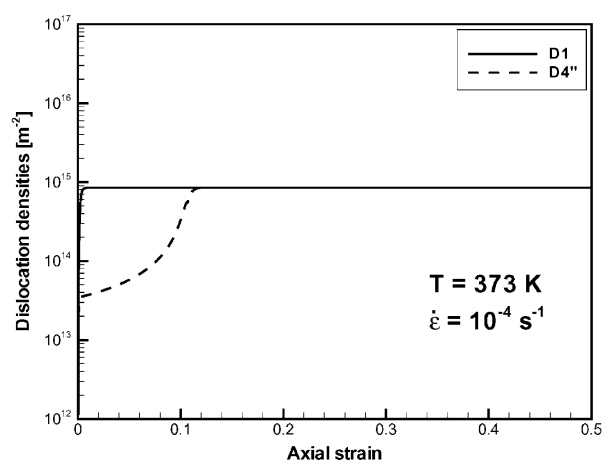

(c) $\dot{\epsilon}=10^{-4} s^{-1}$

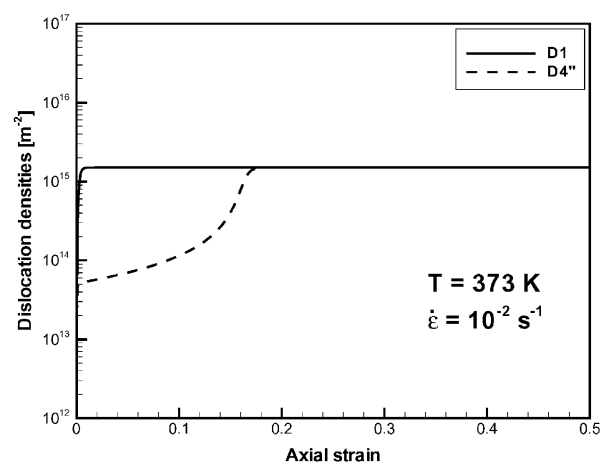

(b) $\dot{\epsilon}=10^{-2} s^{-1}$

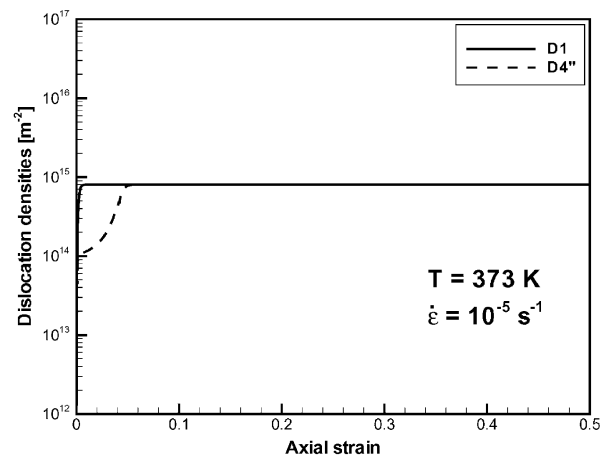

(d) $\dot{\epsilon}=10^{-5} s^{-1}$

Fig. 13. Evolution of dislocation densities as a function of strain-rate $(T=373 \mathrm{~K})$.

characteristic of those orientations. This extreme sensitivity is due to the fact that small deviations from a high-symmetry loading axis, of the order of a degree or less, may result in the activation of a different set of slip systems, which may in turn have a large effect on the hardening rates. This pathological behavior is illustrated in Fig. 15, which shows the stress-strain curve obtained by randomly offsetting by less than one degree the nominal loading axis [101]. The resulting reduction in the hardening rate is quite remarkable. In addition, the experimental curve falls between the bounds of the stressstrain curves for the nominal and perturbed directions. This comparison exemplifies the need to take experimental scatter into account when assessing the fidelity of models, specially where crystals loaded along directions of high symmetry are concerned.

\section{Summary and conclusions}

We have developed a micromechanical model of the hardening, rate-sensitivity and thermal softening of bcc crystals. The model is predicated upon the consideration of 


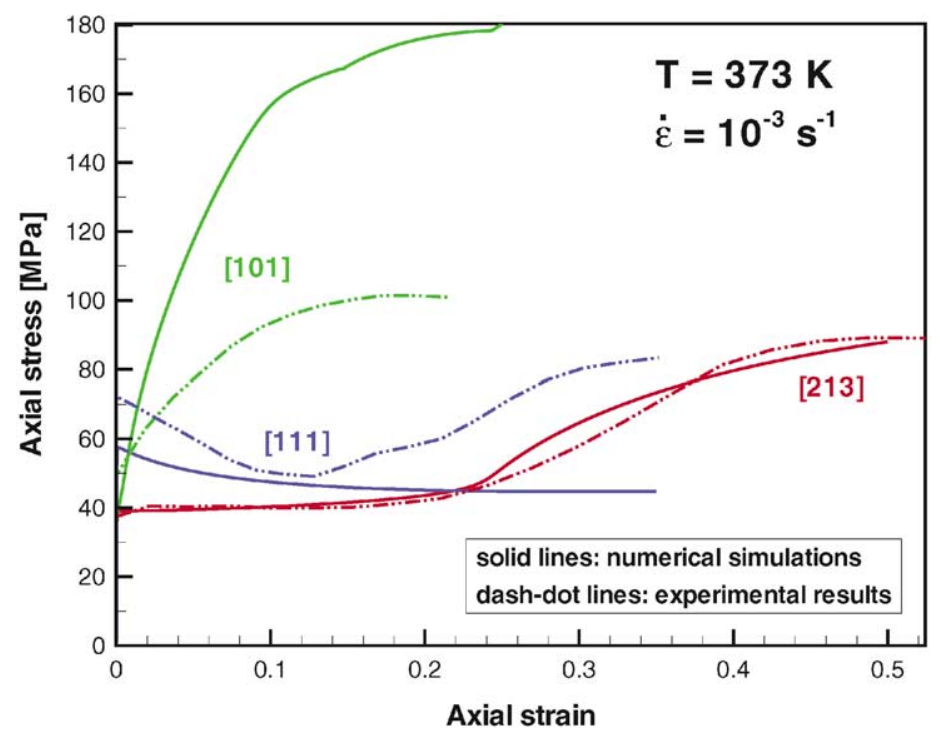

Fig. 14. Orientation dependence of stress-strain curves: comparison with experimental results (Mitchell and Spitzig, 1965).

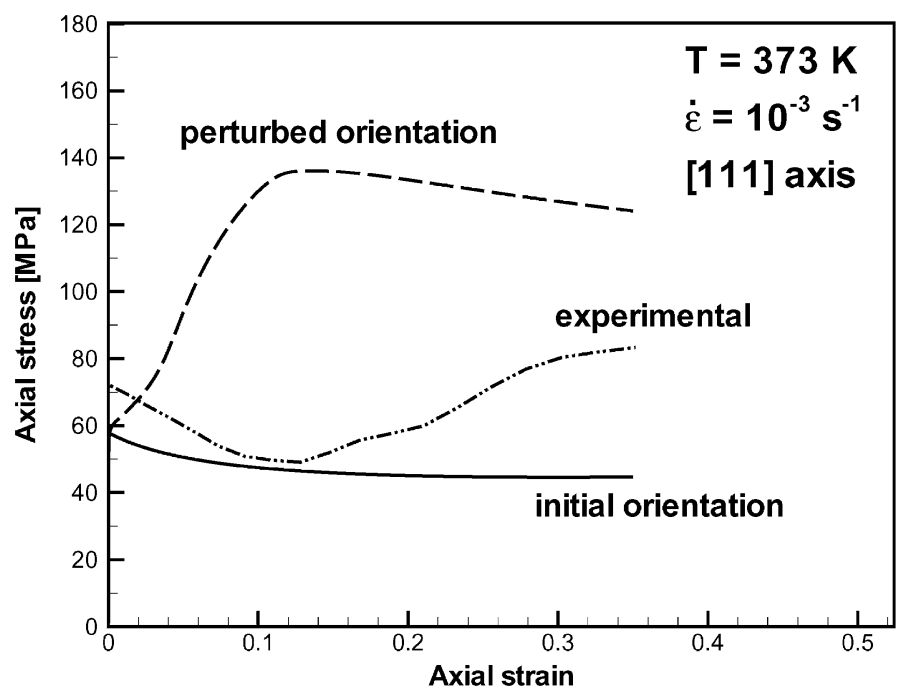

Fig. 15. Effect of a small perturbation in the tensile orientation on the stress-strain curve.

an 'irreducible' set of unit processes, consisting of: double-kink formation and thermally activated motion of kinks; the close-range interactions between primary and forest dislocation, leading to the formation of jogs; the percolation motion of dislocations through a random array of forest dislocations introducing short-range obstacles of different strengths; dislocation multiplication due to breeding by double cross-slip; 
and dislocation pair-annihilation. Each of these processes accounts for-and is needed for matching - salient and clearly recognizable features of the experimental record. In particular, on the basis of detailed comparisons with the experimental data of Mitchell and Spitzig (1965), the model is found to capture: the dependence of the initial yield point on temperature and strain rate; the presence of a marked stage I of easy glide, specially at low temperature and high strain rates; the sharp onset of stage II hardening and its tendency to shift towards lower strains as the temperature increases or the strain rate decreases; the initial parabolic hardening followed by saturation within the stage II of hardening; the temperature and strain-rate dependence of the saturation stress; and the orientation dependence of the hardening rates.

The choice of analysis tools which we have brought to bear on the unit processes of interest, e.g., transition-state theory, stochastic modeling, and simple linear-elastic models of defects and their interactions, is to a large extent conditioned by our desire to derive closed-form analytical expressions for all constitutive relations. As noted throughout the paper, many of the mechanisms under consideration are amenable to a more complete analysis by recourse to atomistic or continuum methods. However, at this stage of development, direct simulation methods, be it atomistic or continuum based, tend to produce unmanageable quantities of numerical data and rarely result in analytical descriptions of effective behavior. The daunting task of post-processing these data sets and uncovering patterns and laws within them which can be given analytical expression is as yet a largely unfulfilled goal of multiscale modeling.

This larger picture notwithstanding, one concrete and workable link between micromechanical models and first-principles calculations concerns the calculation of material constants. A partial list relevant to the present model includes: energy barriers and attempt frequencies for double-kink formation, kink migration, dislocation unpinning, cross-slip, and pair annihilation; dislocation-line and jog energies; and junction strengths. Other properties which have yielded to direct calculation include the volumetric equation of state (EoS), the pressure dependence of yield, and the pressure and temperature dependence of elastic moduli. References to recent work concerned with the calculation of these material properties have been given throughout the paper. As noted earlier, these results provide a suitable basis for future extensions of the present model to higher temperatures, pressures and strain-rates.

\section{Acknowledgements}

The support of the DOE through Caltech's ASCI Center for the Simulation of the Dynamic Response of Materials is gratefully acknowledged. LS also wishes to acknowledge support from the Belgian National Fund for Scientific Research (FNRS).

\section{References}

Arias, T., Joannopoulos, J., 1994. Ab-initio theory of dislocation interactions-from close-range spontaneous annihilation to the long-range continuum-limit. Phys. Rev. Lett. 73 (5), 680-683.

Asaro, R.J., Rice, J.R., 1977. Strain localization in ductile single crystals. J. Mech. Phys. Solids 25, 309. 
Ashby, M.F., 1972. The deformation of plastically non-homogeneous alloys. In: Kelly, A., Nicholson, R.B. (Eds.), Strengthening Methods in Crystals. Wiley, New York.

Baskes, M., 1999. The status role of modeling and simulation in materials science and engineering. Curr. Opinion Solid State Mater. Sci. 4 (3), 273-277.

Baskes, M., Hoagland, R., Tsuji, T., 1998. An atomistic study of the strength of an extended-dislocation barrier. Model. Simulat. Mater. Sci. Eng. 6 (1), 9-18.

Bengus, V., Dolgin, A., Tabachnikova, E., Efimov, Y., 1985. Twinning asymmetry of niobium single-crystals at 4.2 k. Phys. Met. 5 (5), 992-998.

Brailsford, A., 1969. Electronic component of dislocation drag in metals. Phys. Rev. 186, 959-961.

Bulatov, V., Kubin, L., 1998. Dislocation modelling at atomistic and mesoscopic scales. Curr. Opinion Solid State Mater. Sci. 3 (6), 558-561.

Bulatov, V., Richmond, O., Glazov, M., 1999. An atomistic dislocation mechanism of pressure-dependent plastic flow in aluminum. Acta Mater. 47 (12), 3507-3514.

Campbell, G., Foiles, S., Huang, H., Hughes, D., King, W., Lassila, D., Nikkel, D., de la Rubia, T., Shu, J., Smyshlyaev, V., 1998. Multi-scale modeling of polycrystal plasticity: a workshop report. Mater. Sci. Eng. A 251 (1-2), 1-22.

Chang, J., Bulatov, V., Yip, S., 1999. Molecular dynamics study of edge dislocation motion in a bec metal J. Comput. Aided Mater. Design 6 (2-3), 165-173.

Cohen, R., Gulseren, O., Hemley, R., 2000. Accuracy of equation-of-state formulations. Am. Mineral. 85 (2), 338-344.

Cohen, R., Stixrude, L., Wasserman, E., 1997. Tight-binding computations of elastic anisotropy of fe, xe, and si under compression. Phys. Rev. B 56 (14), 8575-8589.

Cuitiño, A.M., Ortiz, M., 1992. Computational modelling of single crystals. Model. Simulat. Mater. Sci. Eng. 1, 255-263.

Cuitiño, A.M., Ortiz, M., 1993. Constitutive modeling of $11_{2}$ intermetallic crystals. Mater. Sci. Eng. A170, $111-123$.

Danna, G., Benoit, W., 1993. Dynamic recovery of the microstructure of screw dislocations in high-purity bcc metals. Mater. Sci. Eng. 164 (1-2), 191-195.

Duesbery, M.S., Vitek, V., Bowen, D.K., 1973. Proc. R. Soc. London A332, 85.

Duesbery, M.S., Vitek, V., 1998. Plastic anisotropy in bcc transition metals. Acta Mater. 46 (5), $1481-1492$.

Duesbery, M.S., Xu, W., 1998. The motion of edge dislocations in body-centered cubic metals. Scr. Mater. 39 (3), 283-287.

Dybiec, H., 1995. Model of the early deformation stage of bcc metals in low-temperature. Z. Metal. 86 (7), 512-517.

Essmann, U., Mughrabi, H., 1979. Annihilation of dislocations during tensile and cyclic deformation and limits of dislocation densities. Philos. Mag. A 40, 731-756.

Essmann, U., Rapp, M., 1973. Slip in copper crystals following weak neutron bombardment. Acta Metal. 21,1305

Foreman, A.J.E., Makin, M.J., 1966. Dislocation movement through random arrays of obstacles. Philos. Mag. $14,911$.

Foreman, A.J.E., Makin, M.J., 1967. Dislocation movement through random arrays of obstacles. Can. J. Phys. 45, 273.

Franciosi, P., Zaoui, A., 1982. Multislip in f.c.c. crystals: a theoretical approach compared with experimental data. Acta Metal. 30, 1627.

Franciosi, P., 1983. Glide mechanisms in b.c.c. crystals: an investigation of the case of $\alpha$-iron through multislip and latent hardening tests. Acta Metal. 31, 1331.

Frank, F., Read, W., 1950. Symposium on Plastic Deformation of Crystalline Solids, Carnegie Institute of Technology, Pittsburgh. Philo. Mag. 79, 722.

Gillis, P.P., Gilman, J., 1965. Dynamical dislocation theory of crystal plasticity. II. Easy glide and strain hardening. J. Appl. Phys. 36, 3380.

Grosskreutz, J.C., Mughrabi, H., 1975. Description of the work-hardened structure at low temperature in cyclic deformation. In: Argon, A.S. (Ed.), Constitutive Equations in Plasticity. MIT Press, Cambridge, MA, pp. 251-326.

Havner, K.S., 1973. On the mechanics of crystalline solids. J. Mech. Phys. Solids 21, 383. 
Hill, R., Rice, J.R., 1972. Constitutive analysis of elastic-plastic crystals at arbitary strains. J. Mech. Phys. Solids 20, 401.

Hirsch, P.B., 1960. Fifth International Conference on Crystallography. Cambridge University, p. 139.

Hirth, J., Hoagland, R., 1993. Nonlinearities in the static energetics and in the kinematics of dislocations. Physica D 66 (1-2), 71-77.

Hirth, J.P., Lothe, J., 1968. Theory of Dislocations. McGraw-Hill, New York.

Huang, H., Ghoniem, N., de la Rubia, T., Rhee, M., Zbib, H., Hirth, J., 1999. Stability of dislocation short-range reactions in bcc crystals. J. Eng. Mater. Technol.-Trans. ASME 121 (2), 143-150.

Hull, D., Bacon, D.J., 1984. Introduction to Dislocations, 3rd Edition, Vol. 37 of International Series on Materials Science and Technology. Elsevier Science Inc., Amsterdam.

Ismail-Beigi, S., Arias, T., 2000. Ab initio study of screw dislocations in mo and ta: a new picture of plasticity in bec transition metals. Phys. Rev. Lett. 84 (7), 1499-1502.

Johnston, W.G., Gilman, J.J., 1959. Dislocation velocities, dislocation densities and plastic flow in lithium fluoride crystals. J Appl. Phys. 30, 129.

Johnston, W.G., Gilman, J.J., 1960. Dislocation multiplication in lithium fluoride crystals. J Appl. Phys. 31, 632.

Kocks, U.F., 1966. A statistical theory of flow stress and work-hardening. Philos. Mag. 13, 541.

Kocks, U.F., Argon, A.S., Ashby, M.F., 1975. In: Chalmers, B., Christian, J.W., Massalski, T.B. (Eds.), Thermodynamics and Kinetics of Slip, Vol. 19 of Progress in Materials Science. Pergamon Press, Oxford.

Koslowski, M., Cuitiño, A.M., Ortiz, M., 2001. A phase-field theory of dislocation dynamics, strain hardening and hysteresis in ductile single crystals at low temperatures. J. Mech. Phys. Solids. Submitted for publication.

Kubin, L.P., Devincre, B., Tang, M., 1998. J. Comput. Aided Mater. Design 5, 31.

Kuhlmann-Wilsdorf, D., 1989. Theory of plastic deformation: properties of low energy dislocation structures. Mater. Sci. Eng. A113, 1.

Lachenmann, R., Schultz, H., 1970. Scr. Metal. 4, 33.

Lagerlof, K., 1993. On deformation twinning in bcc metals. Acta Metal. Mater. 41 (7), 2143-2151.

Lee, E.H., 1969. Elastic-plastic deformation at finite strains. J Appl. Mech. 36, 1.

Lee, Y., Subhash, G., Ravichandran, G., 1999. Constitutive modeling of textured body-centered-cubic (bcc) polycrystals. Int. J. Plastic. 15 (6), 625-645.

Li, J.C.M., 1961. Cross slip and cross climb induced by a locked dislocation. J. Appl. Phys. $32,593$.

Mitchell, T., Spitzig, W., 1965. Three-stage hardening in tantalum single crystals. Acta Metal. 13, 1169-1179.

Moriarty, J., Xu, W., Soderlind, P., Belak, J., Yang, L., Zhu, J., 1999. Atomistic simulations for multiscale modeling in bcc metals. J. Eng. Mater. Technol.-Trans. ASME 121 (2), 120-125.

Mughrabi, H., 1975. Description of the dislocation structure after unidirectional deformation at low temperatures. In: Argon, A. (Ed.), Constitutive Equations in Plasticity. MIT Press, Cambridge, MA, pp. 199-250.

Ortiz, M., 1999. Plastic yielding as a phase transition. J. Appl. Mech.-Trans. ASME 66 (2), $289-298$.

Ortiz, M., Popov, E.P., 1982. A statistical theory of polycrystalline plasticity. Proc. R. Soc. London A379, 439-458.

Ortiz, M., Stainier, L., 1999. The variational formulation of viscoplastic constitutive updates. Comput. Methods Appl. Mech. Eng. 171 (3-4), 419-444.

Phillips, R., 1998. Multiscale modeling in the mechanics of materials. Curr. Opinion Solid State Mater. Sci. 3 (6), 526-532.

Phillips, R., Rodney, D., Shenoy, V., Tadmor, E., Ortiz, M., 1999. Hierarchical models of plasticity: dislocation nucleation and interaction. Model. Simulat. Mater. Sci. Eng. 7 (5), 769-780.

Rao, S., Parthasarathy, T., Woodward, C., 1999. Atomistic simulation of cross-slip processes in model fcc structures. Philos. Mag. A 79 (5), 1167-1192.

Rasmussen, T., Jacobsen, K., Leffers, T., Pedersen, O., Srinivasan, S., Jonsson, H., 1997. Atomistic determination of cross-slip pathway and energetics. Phys. Rev. Lett. 79 (19), 3676-3679.

Rasmussen, T., Vegge, T., Leffers, T., Pedersen, O., Jacobsen, K., 2000. Simulation of structure and annihilation of screw dislocation dipoles. Philos. Mag. A 80 (5), 1273-1290.

Rhee, M., Zbib, H., Hirth, J., Huang, H., de la Rubia, T., 1998. Models for long-/short-range interactions and cross slip in $3 \mathrm{~d}$ dislocation simulation of bcc single crystals. Model. Simulat. Mater. Sci. Eng. 6 (4), 467-492. 
Rice, J.R., 1971. Inelastic constitutive relations for solids: an internal-variable theory and its applications to metal plasticity. J. Mech. Phys. Solids 19, 433.

Rodney, D., Phillips, R., 1999. Structure and strength of dislocation junctions: an atomic level analysis. Phys. Rev. Lett. 82 (8), 1704-1707.

Seeger, A., Hollang, L., 2000. The flow-stress asymmetry of ultra-pure molybdenum single crystals. Mater. Trans. JIM 41 (1), 141-151.

Seeger, A., Schiller, P., 1962. Acta Metal. 10, 348.

Shenoy, V., Kukta, R., Phillips, R., 2000. Mesoscopic analysis of structure and strength of dislocation junctions in fcc metals. Phys. Rev. Lett. 84 (7), 1491-1494.

Simmons, G., Wang, H., 1971. Single crystal elastic constants and calculated aggregate properties: A handbook. M.I.T. Press, Cambridge, MA.

Soderlind, P., Moriarty, J., 1998. First-principles theory of ta up to 10 mbar pressure: structural and mechanical properties. Phys. Rev. B 57 (17), 10340-10350.

Steinle-Neumann, G., Stixrude, L., Cohen, R., 1999. First-principles elastic constants for the hcp transition metals fe, co, and re at high pressure. Phys. Rev. B 60 (2), 791-799.

Suzuki, T., Takeuchi, S., Yoshinaga, H., 1991. Dislocation Dynamics and Plasticity. Springer, Berlin.

Tang, M., Devincre, B., Kubin, L., 1999. Simulation and modelling of forest hardening in body centre cubic crystals at low temperature. Model. Simulat. Mater. Sci. Eng. 7 (5), 893-908.

Tang, M., Kubin, L., Canova, G., 1998. Dislocation mobility and the mechanical response of bcc single crystals: A mesoscopic approach. Acta Mater. 46 (9), 3221-3235.

Teodosiu, C., 1969. A dynamic theory of dislocations and its applications to the theory of the elastic-plastic continuum. In: Simmons, J.A. (Ed.), Conference of the Fundamental Aspects of Dislocation Theory, Vol. 2. Natl. Bureau of Standards Special Publication. Washington D.C., p. 837.

Vitek, V., 1976. Proc. R. Soc. London A352, 109.

Vitek, V., 1992. Structure of dislocation cores in metallic materials and its impact on their plastic behavior. Prog. Mater. Sci. 36, 1-27.

Wang, G., Strachan, A., Cagin, T., Goddard, W.I., 2000. Molecular dynamics simulations of $1 / 2 \mathrm{a}\langle 111\rangle$ screw dislocation in ta. Mater. Sci. Eng. A-stuct 309, 133-137.

Wasserbäch, W., 1986. Philos. Mag. A53, 335.

Wasserman, E., Stixrude, L., Cohen, R., 1996. Thermal properties of iron at high pressures and temperatures. Phys. Rev. B 53 (13), 8296-8309.

Weiner, J.H., 1983. Statistical Mechanics of Elasticity. Wiley, New York.

$\mathrm{Xu}$, W., Moriarty, J., 1996. Atomistic simulation of ideal shear strength, point defects, and screw dislocations in bcc transition metals: Mo as a prototype. Phys. Rev. B 54 (10), 6941-6951.

Xu, W., Moriarty, J., 1998. Accurate atomistic simulations of the peierls barrier and kink-pair formation energy for $\langle 111\rangle$ screw dislocations in bcc mo. Comput. Mater. Sci. 9 (3-4), 348-356.

Zbib, H., de la Rubia, T., Rhee, M., Hirth, J., 2000. 3d dislocation dynamics: stress-strain behavior and hardening mechanisms in fcc and bcc metals. J. Nucl. Mater. 276, 154-165. 